\title{
Features of collisionless turbulence in the intracluster medium from simulated Faraday Rotation maps
}

\author{
M. S. Nakwacki, ${ }^{1,2 \star}$ G. Kowal, ${ }^{3,4 \star}$ R. Santos-Lima, ${ }^{5 \star}$ E. M. de Gouveia Dal Pino ${ }^{5}$ \\ and D. A. Falceta-Gonçalves ${ }^{3,6}$ \\ ${ }^{1}$ Instituto de Astronomía y Física del Espacio, UBA-CONICET, Argentina \\ ${ }^{2}$ Facultad de Ciencias Exactas y Naturales, Universidad de Buenos Aires, Argentina \\ ${ }^{3}$ Escola de Artes, Ciências e Humanidades, Universidade de São Paulo, São Paulo, SP, Brazil \\ ${ }^{4}$ Núcleo de Astrofsica Terica, Universidade Cruzeiro do Sul, São Paulo, SP, Brazil \\ ${ }^{5}$ Instituto de Astronomía, Geofísica e Ciências Atmosféricas, Universidade de São Paulo, Brazil \\ ${ }^{6}$ SUPA, School of Physics \& Astronomy, University of St Andrews, St Andrews, Fife KY16 9SS, UK
}

Accepted 2015 November 2. Received 2015 November 2; in original form 2015 May 25

\begin{abstract}
Observations of the intracluster medium (ICM) in galaxy clusters suggest for the presence of turbulence and the magnetic fields' existence has been proved through observations of Faraday Rotation (FR) and synchrotron emission. The ICM is also known to be filled by a rarefied weakly collisional plasma. In this work, we study the possible signatures left on FR maps by collisionless instabilities. For this purpose, we use a numerical approach to investigate the dynamics of the turbulence in collisionless plasmas based on an magnetohydrodynamical (MHD) formalism taking into account different levels of pressure anisotropy. We consider models covering the sub/super-Alfvénic and trans/supersonic regimes, one of them representing the fiducial conditions corresponding to the ICM. From the simulated models, we compute FR maps and analyse several statistical indicators in order to characterize the magnetic field structure and compare the results obtained with the collisionless model to those obtained using standard collisional MHD framework. We find that important imprints of the pressure anisotropy prevails in the magnetic field and also manifest in the associated FR maps which evidence smaller correlation lengths in the collisionless MHD case. These points are remarkably noticeable for the case mimicking the conditions prevailing in ICM. Nevertheless, in this study we have neglected the decrease of pressure anisotropy due to the feedback of the instabilities that naturally arise in collisionless plasmas at small scales. This decrease may not affect the statistical imprint differences described above, but should be examined elsewhere.
\end{abstract}

Key words: magnetic fields - turbulence-methods: numerical-galaxies: clusters: intracluster medium.

\section{INTRODUCTION}

The intracluster medium (ICM) in galaxy clusters is a very dynamic environment. Galaxy clusters are built up by gravitational mergers of smaller units according to the standard scenario of structure formation. They are composed of hundreds of galaxies in Mpc scale, being the largest viralized structures $\left(\approx 10^{14}-10^{15} \mathrm{M}_{\odot}\right)$ in the Universe. Only $\approx 15$ per cent of the total mass in galaxy clusters corresponds to baryonic matter, being most of it ( $>80$ per cent) in the ICM, and only a small fraction ( $<20$ per cent) in stars (Gonzalez, Zaritsky \& Zabludoff 2007). The ICM is filled with a hot (at the

^E-mail: sole@agro.uba.ar (MSN); grzegorz.kowal@usp.br (GK); rlima@ astro.iag.usp.br (RS-L) virial temperature, $10^{7}-10^{8} \mathrm{~K}$ ) and rarefied gas emitting in the soft$\mathrm{X}$-ray domain through optically thin bremsstrahlung. Its typical number density ranges from 0.1 to $0.001 \mathrm{~cm}^{-3}$ (Banerjee \& Sharma 2014, and references therein).

Modelling of such dynamic environment requires adequate knowledge of the role of the non-thermal components, namely the relativistic particles and the magnetic fields. The presence of magnetic fields has been revealed by the study of the synchrotron emission from diffuse radio sources in the ICM (radio haloes and relics), the Faraday Rotation (FR) of the synchrotron emission from radio sources embedded and behind the cluster (see Ferrari et al. 2008; Feretti et al. 2012; Brunetti \& Jones 2014). Through these observations, it is possible to constrain the main properties of the magnetic fields and to understand the physical processes taking place in the ICM (Ferrari et al. 2008; Bonafede et al. 2010b; Brüggen et al. 2012; 
Brunetti \& Jones 2014; Dolag et al. 2005). For example, radio observations have discovered the presence of radio emission arising from the ICM and not connected to the emission of the individual galaxies in the clusters. These radio sources are called radio haloes, radio relics and mini haloes, depending on their position and observational properties. The emission mechanism is synchrotron from ultra relativistic electrons diffusing in a turbulent magnetic field at $\mu \mathrm{G}$ level. Consequently, a precise knowledge of the ICM magnetic fields could be an important tool to clarify the origin of the relativistic particles which are responsible for the synchrotron diffuse radio haloes and relics detected in several galaxy clusters. In particular, observations suggest that radio relics host relatively large magnetic fields (at $\mu \mathrm{G}$ level; Markevitch et al. 2005; van Weeren et al. 2011). This magnitude of magnetic fields is typical in the central regions of clusters, but is not expected near the periphery, where relics are located.

Several mechanisms have been studied in connection with the amplification of magnetic fields (Dolag, Bartelmann \& Lesch 2002; Brüggen et al. 2005; Subramanian, Shukurov \& Haugen 2006; Iapichino \& Brüggen 2012). A recent work of Brüggen (2013) has studied the amplification of magnetic fields due to shocks including diffusion of cosmic rays, while Santos-Lima et al. (2014) and Falceta-Gonçalves \& Kowal (2015) have taken into account the pressure anisotropy of the plasma, thus including the presence of instabilities and turbulent dynamo to characterize the amplification of the magnetic field. The impact of such magnetic fields on the FR of the synchrotron emission is studied in Brandenburg \& Stepanov (2014), Sofue, Fujimoto \& Wielebinski (1986), Beck et al. (1996), Fletcher (2010) and Beck \& Wielebinski (2013). In particular, Brandenburg \& Stepanov (2014) showed that a helical magnetic field with an appropriate sign of helicity can compensate the so-called Faraday depolarization, which can have important observational consequences.

Magnetic fields in the ICM also affect the thermal conduction (see Narayan \& Medvedev 2001; Lazarian 2006), given the typical values of the thermal electron gyro-radius $\left(\approx 10^{8} \mathrm{~cm}\right.$ for $T=10^{8} \mathrm{~K}$ and $B=1 \mu \mathrm{G})$ are much smaller than any scale of interest in clusters, and in particular than the particle mean free path due to collisions (e.g. 0.05-30 kpc for Hydra A cluster, 3-7 kpc for Coma cluster; see e.g. Schekochihin \& Cowley 2006; Andrade-Santos et al. 2013; Sanders et al. 2013). It follows that the effective mean free path for diffusion perpendicular to the magnetic field lines is reduced, and being the magnetic field tangled in the ICM, it is crucial to obtain information about the magnetic field coherence length (Nakwacki \& Peralta-Ramos 2012; Kunz 2011; Santos-Lima et al. 2014). Magnetic fields in the ICM are also important for the dynamics of cosmic rays, their diffusion and acceleration (see e.g. Berezinsky, Blasi \& Ptuskin 1997; Petrosian 2001; Brunetti \& Lazarian 2007).

Usually, the features and phenomena listed above are studied in the context of standard collisional magnetohydrodynamics. However, the ICM is magnetized and nearly collisionless, i.e. the gyrofrequency of the ions is greater than that of binary collision. Plasmas with such characteristics are known to develop anisotropic pressures with respect to the magnetic field orientation (see e.g. Quest \& Shapiro 1996; Barakat \& Schunk 1982; Krall \& Trivelpiece 1973), whose imprints may survive for considerably long periods compared with the dynamical time-scales of the system (Kowal, Falceta-Gonçalves \& Lazarian 2011). The pressure anisotropy can give rise to instabilities which are not present in the isotropic case, namely, firehose and mirror instabilities. In the fluid scales, these have a deep impact on the evolution of turbulence and magnetic field geometry.
In a recent work, Santos-Lima et al. (2014) have studied the amplification of magnetic field in the ICM considering the presence of firehose and mirror instabilities caused by the anisotropic pressure tensor, but including a plausible model for anisotropy relaxation to mimic the effect of scattering of individual ions by fluctuations induced by these plasma instabilities in the kinetic scales. They found that anisotropy in the collisionless fluid is naturally created by turbulent motions due to fluctuations of magnetic field and gas density, but the fluctuations of the magnetic field in large scales are mostly suppressed. The inclusion of such modelling for anisotropy relaxation has been also considered by Mogavero \& Schekochihin (2014), who found that the amplification of seed fields is reduced with the use of pressure anisotropy relaxation.

Improving our theoretical understanding of the dynamics of the ICM plasma can lead to clearer interpretation of observational data with the aim of characterizing the magnetic field in the ICM. In particular, numerical simulations are a useful tool because they allow a control over the free parameters that are usually considered in observational models. In this respect, the purpose of this work is twofold. On one hand, we try to give a comprehensive analysis of numerical simulations of turbulent plasma under different conditions, including those prevailing in the ICM, taking into account the effects of pressure anisotropy, and compare the results with those obtained in the isotropic (magnetohydrodynamical (MHD)) case. On the other hand, we attempt to provide a connection between the properties of the magnetic field of the ICM that arise using collisionless and collisional MHD simulations and those usually assumed in observational studies. In particular, we will consider FR maps and their statistical properties.

In order to determine the influence of pressure anisotropy on the turbulent evolution of the magnetic field in the ICM and on the corresponding rotation maps, in this work we use a collisionless magnetohydrodynamic formalism with a double-isothermal closure as implemented in Kowal et al. (2011). Considering that in the ICM the cyclotron frequency is much larger than the collision frequency, the authors have studied different processes related to pressure anisotropy. In particular, they used different plasma configurations in order to determine the appearance of mirror/firehose instabilities and the implications for the statistics in the velocity field and density (Kowal et al. 2011). Here, we do not consider the effects of relaxation of the pressure anisotropy due to the instabilities feedback upon the plasma as in Santos-Lima et al. (2014), but will leave this analysis to a forthcoming work.

This paper is organized as follows. In Section 2, we give a brief overview of FR maps and their application to study the magnetic field structure in the ICM through relevant statistical indicators. In Section 3, we describe the theoretical setup, followed by the main results, presented in Section 4 and the discussions, in Section 5. Finally, in Section 6 we summarize our results and draw the main conclusions.

\section{FARADAY ROTATION MAPS OF GALAXY CLUSTERS}

As mentioned in the previous section, polarization observations of synchrotron emission have become an important diagnostic tool in the study of the extragalactic magnetic fields. A brief explanation of this effect is as follows. When linearly polarized waves, as synchrotron radiation, propagate through a magneto-ionic medium as the ICM, its polarization properties change. Due to the birefringence of the medium, in fact, the polarization plane of the radiation is rotated as a function of frequency. This effect is the 
so-called FR. If we indicate the intrinsic polarization angle as $\Phi_{\text {int }}$, the effect of the FR can be parametrized as $\Phi_{\text {obs }}=\Phi_{\text {int }}+R M \lambda^{2}$, with the rotation measure (RM) defined as

$\mathrm{RM}=812 \int_{0}^{L} n_{\mathrm{e}} B_{\|} \mathrm{d} l \quad$ in $\left(\operatorname{rad~m}^{-2}\right)$

with the magnetic field $B_{\|}$in $\mu \mathrm{G}$, the ambient electronic density $n_{\mathrm{e}}$ in $\mathrm{cm}^{-3}$, both along the line of sight ( $\left.\mathrm{LoS}\right)$, and the distance to the source $L$ in kpc.

Radio observations of the FR have revealed important features of the magnetic field, e.g. patchy structures, over a large range of spatial scales (see e.g. Clarke, Kronberg \& Böhringer 2001; Carilli \& Taylor 2002; Murgia et al. 2004; Govoni \& Feretti 2004; Vogt \& Enßlin 2005; Govoni et al. 2010; Bonafede et al. 2010b, 2011; Kuchar \& Enßlin 2011). The random magnetic field must be both tangled on small scales as observed in the RM images and also fluctuate on scales one or even 2 orders of magnitude larger, which account for the large-scale turbulence. For this reason, it is necessary to consider cluster magnetic field models where both small- and large-scale structures coexist. This behaviour has been studied theoretically on a statistical basis in previous works. Murgia et al. (2004) have considered a magnetic fluctuation spectrum in the form:

$\left|B_{k}\right|^{2} \propto C_{n}^{2} k^{-n}$

where $n$ represents the spectral index to be constrained by observations and/or numerical simulations and $C_{n}^{2}$ is the power spectrum normalization. The power spectrum described in equation (2) has been used in several works proposing a particular spectral index to reproduce synthetic FR maps, which usually lies in the range 2-4 (see e.g. Murgia et al. 2004; Bonafede et al. 2010b), depending on the assumed $B-n$ correlation law.

The origin of these magnetic fields is unknown, but possibly amplified during the formation of galaxy clusters (e.g. Dolag 2006; de Gouveia Dal Pino 2010, for reviews). Bhat \& Subramanian (2013) considered a fluctuation dynamo action in a turbulent medium to study the FR measure from the radio emission of background sources seen through the intermittent magnetic field generated by the dynamo. These authors showed that even though the magnetic field generated is intermittent, it still allows the contribution to FR measure to be significant. In this sense, FR measurements are crucial to infer the presence of coherent magnetic fields and to probe the distribution of the spectral power over different scales.

In order to track the collisionless effects, we investigate the statistical properties of the FR maps individually. For this purpose, we employ statistical tools like the probability density function (PDF) and the power spectrum of the FR maps, aiming at analysing these maps for different regimes of turbulence, always comparing with the output from collisional MHD models. We will also present the autocorrelation function of the synthetic FR maps, which is more directly connected with observations.

\section{THEORETICAL SETUP AND NUMERICAL SIMULATIONS}

\subsection{Double-isothermal collisionless MHD approximation}

The double-isothermal collisionless MHD approximation consists of a single fluid plasma with the pressure replaced by the pressure tensor described by two isothermal equation of states with independent isothermal sound speeds along the parallel and perpendicular direction to the local magnetic field. The conservation laws which describe this approximation (e.g. Kowal et al. 2011) ${ }^{1}$ are:

$\frac{\partial \rho}{\partial t}+\nabla \cdot(\rho \boldsymbol{v})=0$

$\frac{\partial(\rho \boldsymbol{v})}{\partial t}+\nabla \cdot\left[\rho \boldsymbol{v} \boldsymbol{v}+\left(\mathbf{P}+\frac{B^{2}}{8 \pi}\right) \mathbf{I}-\frac{\boldsymbol{B} \boldsymbol{B}}{4 \pi}\right]=\boldsymbol{f}$,

$\frac{\partial \boldsymbol{B}}{\partial t}-\nabla \times(\boldsymbol{v} \times \boldsymbol{B})=0$,

where the pressure tensor is described by two components:

$\mathbf{P}=p_{\perp} \mathbf{I}+\left(p_{\|}-p_{\perp}\right) \hat{b} \hat{b}, \quad p_{\perp}=c_{\perp}^{2} \rho \quad$ and $\quad p_{\|}=c_{\|}^{2} \rho$,

with $\hat{b}=\boldsymbol{B} /|\boldsymbol{B}|$, and $c_{\|, \perp}$ being the sound speeds parallel and perpendicular to the magnetic field $\boldsymbol{B}$, respectively. Then, the momentum conservation equation can be written as

$\frac{\partial(\rho \boldsymbol{v})}{\partial t}+\nabla \cdot\left[\rho \boldsymbol{v} \boldsymbol{v}+\left(c_{\perp}^{2} \rho+\frac{B^{2}}{8 \pi}\right) \mathbf{I}-(1-\alpha) \boldsymbol{B} \boldsymbol{B}\right]=\boldsymbol{f}$,

where $\alpha=\frac{1}{2}\left(p_{\|}-p_{\perp}\right) / p_{\mathrm{m}}$, and $p_{\mathrm{m}}=B^{2} / 8 \pi$ is the magnetic pressure.

A linear analysis (see Hau \& Wang 2007; Kowal et al. 2011) provides the following dispersion relation for the waves (of wavenumber $k$ and frequency $\omega$ ):

$$
\begin{aligned}
& \left(\frac{\omega}{k}\right)_{\mathrm{A}}^{2}=\left[V_{\mathrm{A}}^{2}-\left(c_{\|}^{2}-c_{\perp}^{2}\right)\right] \cos ^{2} \theta, \\
& \left(\frac{\omega}{k}\right)_{\mathrm{F}, \mathrm{S}}^{2}=\frac{1}{2}\left(c_{\perp}^{2}+V_{\mathrm{A}}^{2} \pm \sqrt{\Delta}\right),
\end{aligned}
$$

where

$$
\begin{aligned}
\Delta= & \left(c_{\perp}^{2}+V_{\mathrm{A}}^{2}\right)^{2}-4\left[c_{\perp}^{2}\left(c_{\|}^{2}-c_{\perp}^{2}\right)+c_{\|}^{2} V_{\mathrm{A}}^{2}\right] \cos ^{2} \theta \\
& +4\left(c_{\|}^{4}-c_{\perp}^{4}\right) \cos ^{4} \theta,
\end{aligned}
$$

and the subscripts A, F and S stand for the Alfvén, fast and slow modes, respectively, in analogy to the standard MHD case. It can be shown that $\Delta>0$ always. The usual MHD dispersion relations are recovered for $c_{\perp}=c_{\|}$.

The first thing to note in the equations above is that now the linear Alfvén wave depends also on the thermal speeds. Also the maximum and minimum values of the fast mode change and can be larger than in the case of isotropic pressure. As the function $\Delta$ is not anymore linear in $\cos ^{2} \theta$, the maximum and minimum speeds of the fast mode can be now between the extremes $\theta=0$ or $\theta=\pi$. The same applies to the maximum speed of the slow modes. Another difference introduced by the anisotropy is the possibility of anomalous slow modes with $\delta b_{\|} \delta \rho>0$, that is, with a positive correlation between the density fluctuations and the magnetic field component parallel to the background magnetic field (see Hau \& Wang 2007 and Appendix B).

\footnotetext{
${ }^{1}$ We note that there are other approaches to collisionless MHD, of which the so-called Chew-Golberger-Low closure (CGL) (Chew, Goldberger \& Low 1956) is the most employed. The main difference between the CGL and the present double-isothermal approach is that the first combines the adiabatic conservation of magnetic momentum of the particles with local thermal energy conservation (that is, the conservation of the local entropy) while the second keeps constant temperatures of the gyrotropic distribution of particle velocities in parallel and perpendicular directions to the local magnetic field (see Kowal et al. 2011).
} 
The most remarkable difference introduced by the anisotropy is the possibility of occurrence of instabilities. When $c_{\|} / c_{\perp}>1$ the firehose instability can arise which tends to bend the magnetic field lines and trap gas within regions of high intensity field. On the other hand, when $c_{\|} / c_{\perp}<1$ the mirror instability can occur pushing gas to regions of smaller magnetic field strength (e.g. Kulsrud 1983). A brief description of the conditions for each instability is given in Appendix A.

\subsection{The numerical code}

In order to study the magnetic field dynamics in the ICM we simulate turbulence in a three-dimensional periodic Cartesian box evolving the double-isothermal collisionless MHD equations (3)-(6). The turbulence is introduced through a source term $f$ on the right-hand side of equations (4) and (7) and is continuously driven at the wave scale $k=2.5$ (which gives an injection scale in the model 2.5 times smaller than the size of the computational box). Our forcing is done in Fourier space in such a way, that the forcing components have random phases at each step and the correlation between them and velocity is removed. This assures that the velocity field is not correlated with our forcing at any temporal or spatial scale. Additionally, our forcing is incompressible, and therefore does not generate density fluctuations by itself. Any compression appearing in the studied systems is the result of the magnetosonic wave interactions or kinetic instabilities developed during the system evolution (see e.g. Kowal, Lazarian \& Beresnyak 2007; Kowal \& Lazarian 2010, and references therein). As turbulence evolves, the MHD modes interact and generate both compressive and incompressive components at scales smaller than the injection one. Therefore, turbulence observed in our models is actually mixture of both modes.

To solve equations (3)-(6), we employ the shock-capturing, second-order Godunov code (see Kowal et al. 2007, 2009; Kowal \& Lazarian 2010; Kowal et al. 2011, for more detailed code description). We do not take into account viscosity or diffusion in the equations. The scale at which the dissipation starts to act is defined by the numerical diffusivity of the scheme (see Section 5.1).

The variables are normalized in code units therefore they can be rescaled to any system by defining three representative quantities from which all the other ones can be derived: the length-scale $L$ (which is given by the computational box size), the gas density $\rho_{0}$ (given by the initial ambient density of the system) and the Alfvén speed defining the time unit $L / V_{\mathrm{A}}$. With this normalization, the isothermal sound speeds $\left(c_{\mathrm{S}}, c_{\|}\right.$and $\left.c_{\perp}\right)$ are also given in units of $V_{\mathrm{A}}$ and the magnetic field has units $V_{\mathrm{A}} \sqrt{4 \pi \rho_{0}}$.

\subsection{Initial conditions}

In order to understand better the connection between some features of the magnetic field and the FR maps, it is useful to consider different initial conditions for the simulations, corresponding to different regimes in which the turbulent plasma evolves. Specifically, we shall analyse the impact of pressure anisotropy on the maps by considering different initial conditions for the magnetic field strength, and parallel/perpendicular pressure anisotropy.

We consider six different models that cover the sub/superAlfvénic and the trans/supersonic regimes; the respective parameters are shown in Table 1 . The turbulent velocity at the injection scale is $V_{\text {turb }} \approx 0.8$. It must be remarked that the classifications of sub/super-Alfvénic and trans/supersonic refer to the collisional MHD models (which are employed for comparison with the collisionless models). These are based on the comparison between $V_{\text {turb }}$
Table 1. Parameters of the turbulent isothermal collisional and double-isothermal collisionless MHD models simulated with the resolution $512^{3}$. The initial density for all models was set to 1.0. For collisional models, the parallel and perpendicular sound speeds are equal, i.e. $c_{\|}=c_{\perp}=c_{\text {snd }}$. Their names indicate the corresponding collisionless models for which both $B_{\text {ext }}$ and $c_{\|}$are the same.

\begin{tabular}{ccccl}
\hline Model & $B_{\text {ext }}$ & $c_{\|}$ & $c_{\perp}$ & $c_{\|}^{2} / c_{\perp}^{2}$ \\
\hline \multicolumn{5}{c}{ Collisional models } \\
$1-2$ & 1.0 & 1.0 & 1.0 & \\
$3-4$ & 0.1 & 0.1 & 0.1 & 1.0 \\
5 & 0.1 & 1.0 & 1.0 & 1.0 \\
6 & 1.0 & 0.1 & 0.1 & 1.0 \\
& & Collisionless models & \\
& 1.0 & 1.0 & 2.0 & \\
1 & 1.0 & 1.0 & 0.5 & 4.0 \\
2 & 0.1 & 0.1 & 0.2 & 0.25 \\
3 & 0.1 & 0.1 & 0.05 & 4.0 \\
4 & 0.1 & 1.0 & 0.5 & 4.0 \\
5 & 1.0 & 0.1 & 0.2 & 0.25 \\
6 & & &
\end{tabular}

and the Alfvén speed $V_{\mathrm{A}}=B_{\text {ext }} / \sqrt{\rho_{0}}$ (in code units), and $V_{\text {turb }}$ and the parallel sound speed $c_{\|}$, respectively. Indeed, as discussed in Section 3.1, an anisotropic 'super-Alfvénic' $\left(V_{\text {turb }}>V_{\mathrm{A}}\right)$ model can be effectively sub-Alfvénic depending on the sound speed. The comparison of the results obtained from these models allows us to get a deeper insight into the effect of the magnetic field topology on the FR maps. We consider the initial magnetic field in the $\hat{x}$ direction, and an initial constant density $\rho_{0}$ set to 1.0 in code units for all six models. We evolve up to $t=5.0$ in code units, when the turbulence is fully developed (we note that the turbulence turn-over time is $\sim 0.4$ in code units).

Fig. B1 in Appendix B shows the linear phase speeds (equation 8) for the initial condition of each of the models in Table 1. It compares the wave speeds of the models with anisotropic pressure with those of isotropic (collisional) MHD models. It highlights, for example, the differences in the effective Alfvén speeds (which also reflect the magnetic field tension). Fig. B1 also reveals what models are initially unstable to the firehose and mirror instabilities. Table 2 shows the magnetic field intensity which is required for each model to develop firehose or mirror unstable regions (see instability conditions in Appendix A). It also classifies the turbulence as super- or sub-Alfvénic and trans- or subsonic according to the corresponding collisional MHD model that is used for comparison with each collisionless model. The last column shows the effective Alfvén speed, that is, the phase speed of the linear Alfvén wave for the initial conditions.

As indicated in Table 1, six collisionless MHD models are considered. Models 1 and 2 are in the transonic and sub-Alfvénic regime. Model 1 is initially unstable to mirror modes (see Table 2 and Fig. B1). Model 2 is initially stable to both mirror and firehose instabilities, but later on, in locations where the magnetic field is reduced to values smaller than the threshold (Table 2), it can develop the firehose instability. Fig. B1 shows that the effective behaviour of Model 2 is super-Alfvénic as the initial effective Alfvén speed is reduced with respect to $V_{A}$. Models 3 and 4 correspond to supersonic and super-Alfvénic regimes. Model 3 is also unstable to mirror modes, and Model 4 can also later develop flow regions which are unstable to the firehose instabilities, if the magnetic field intensity is reduced to values smaller than the threshold for its triggering (Table 2). Model 5 is initially in a transonic and super-Alfvénic 
Table 2. Characteristics of the simulated models from Table 1. In the first and second columns, we show the model name and its mean magnetic field strength. The third and fourth columns show the local magnetic field intensity required to develop the mirror or firehose instabilities, respectively. The next column shows the effective Alfvén speed for the initial conditions of the collisionless models $\left(V_{\mathrm{A} 0, \mathrm{eff}}^{2}=V_{\mathrm{A} 0}^{2}+c_{\perp}^{2}-c_{\|}^{2}\right)$. The last two columns show the sonic and Alfvénic regimes of the developed turbulence.

\begin{tabular}{ccccccc}
\hline Model & $B_{\mathrm{ext}}$ & Mirror & Firehose & $V_{\mathrm{A} 0 \text {,eff }}$ & Sonic regime & Alfvénic regime \\
\hline 1 & 1.0 & $B<3.4$ & - & 2.0 & Trans & Sub \\
2 & 1.0 & - & $B<0.86$ & 0.5 & Trans & Sub \\
3 & 0.1 & $B<0.34$ & - & 0.2 & Super & Sub \\
4 & 0.1 & - & $B<0.086$ & 0.05 & Super & Super \\
5 & 0.1 & - & $B<0.86$ & - & Trans & Super \\
6 & 1.0 & $B<0.34$ & - & Super & Sub \\
\hline
\end{tabular}

turbulence regime and can eventually develop firehose instabilities in regions where the magnetic field is reduced to values below the threshold for this model (see Table 2). This model may represent the physical regime prevailing in compressed zones of the ICM. ${ }^{2}$ Finally, Model 6 is initially in the supersonic and sub-Alfvénic turbulent regime and is stable to both mirror and firehose instabilities. We also note that since this model is strongly sub-Alfvénic, it is very unlikely that later on it will be able to produce magnetic fields as small as those required for the onset of the mirror instability (see Table 2). Besides, the plasma $\beta=p / p_{\mathrm{m}}$ ratio is very low in this case so that the pressure anisotropy will have little dynamical importance on the flow.

The collisional MHD models which are considered for comparison with the collisionless counterparts are also listed in Table 1. They have four different combinations between initial magnetic field strength and sound speed in order fulfil the same initial conditions of the models mentioned above.

We should note that in the ICM the density and temperature profiles are not constant and decay with the distance from the cluster core. Following the model of gas distribution in the relaxed ICM by Cavaliere \& Fusco-Femiano (1978), the density can drop by factor 10-100 at distance of 5 core radii. Similar models are being studied for the pressure profiles providing comparable decay (see Lapi, Cavaliere \& Fusco-Femiano 2012). As the result, the sound speed should not change by more than a factor 2-3 at the distance of 1-2 Mpc from the core. While the profiles for density and temperature can be obtained from the X-ray observations, the profile for large-scale component of magnetic field is poorly know. The problem comes from the fact that in order to obtain the magnetic field strength from RM observations, one has to know the profile of density, which is obtained from the surface density using the mentioned models.

Our numerical models represent local simulation of a box embedded in the ICM far from local sources with its size corresponding to a fraction of the cluster sizes, of the order of tens of kpcs compared to the size of clusters of 1-2 Mpc. It means, that the change of global profiles is small within our numerical domain. In addition, we assume that turbulence is driven at scales larger than our simulation box. These two assumptions justify the use of periodic boundary conditions. We are planning, however, to extend our studies in the future by introducing external profiles for density, pressure and magnetic field, which would also require inclusion of the gravita-

\footnotetext{
${ }^{2}$ We note that Santos-Lima et al. (2014) examined the conditions of the turbulent collisionless plasma in the ICM and found that the flow tends to develop regions predominantly with $c_{\perp}>c_{\|}$(which favours the triggering of mirror instabilities) and compressed regions with $c_{\|}>c_{\perp}$ (which favours the onset of the firehose instability).
}

tional potential of the cluster and better suited boundary conditions, such as hydrostatic ones.

The physical characteristics of all models are discussed below where we present our results in connection with the imprints left on FR maps (Section 4).

\section{RESULTS}

In this section, we present the results obtained from the simulations with parameters given in Table 1 (Models 1-6). In order to extract the main features that the anisotropy imprints on the plasma, we analyse the magnetic field intensity, its probability distribution and energy power spectrum. We present the synthetic FR maps, and perform a detailed statistical analysis focusing on the characteristics of their distributions and power spectra. To complete our analysis, we also present the autocorrelation functions for these maps, which can be compared directly with observations.

Through this section, all presented results consider only the last snapshot corresponding to the time of the simulation $t=5.0$, when the turbulent cascade has been already fully developed.

\subsection{Magnetic field intensity and the role of anisotropy}

Figs 1-4 depict a cut of the magnetic field intensity in the centre of the computational domain for all six models from Table 1 . In these figures we compare the MHD models with isotropic (top rows) and anisotropic pressure (middle and/or bottom rows).

In the case of the transonic and sub-Alfvénic regime, corresponding to Model 1 (second row of Fig. 1), the presence of mirror instability produces strong fluctuations of magnetic field in small scales, which can grow since the turbulent motions are slow compared to the instability growth rate (see Fig. B1). The instability presence is seen in large differences between the magnetic field structure obtained in Model 1 and the corresponding model with the isotropic pressure (seen in the top row of Fig. 1). As pointed out in Kowal et al. (2011), due to the instability growth rate larger at small scales, the instability creates more granulated maps, thus the magnetic field intensity for Model 1 results in a more wrinkled distribution. ${ }^{3}$ The more intense magnetic field regions are distributed in smaller patches. Moreover, the fragmented structures seem to be more aligned with the initial magnetic field.

The transonic and sub-Alfvénic turbulent Model 2 is shown in the bottom row of Fig. 1. Even though this model has an anisotropic stress smaller than the magnetic pressure (i.e. $\left|p_{\|}-p_{\perp}\right| / 2 p_{\mathrm{m}}<1$ )

\footnotetext{
${ }^{3}$ In our numerical simulations, the numerical dissipation suppresses the instabilities in the dissipation range (see Section 5.1).
} 

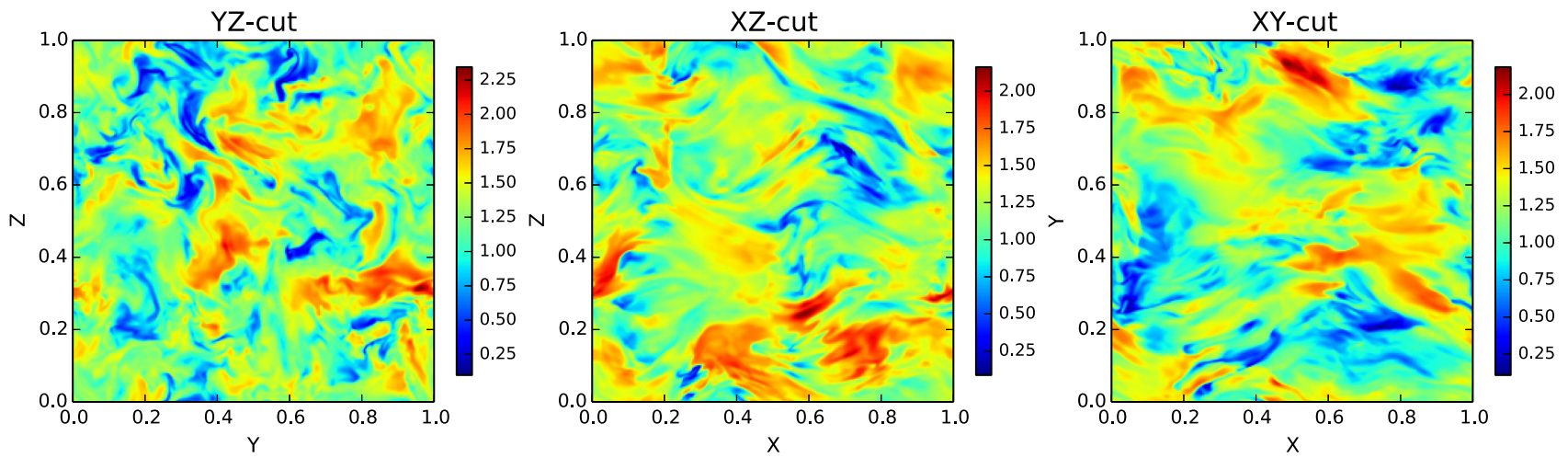

$|\mathrm{B}|$ Cuts - Model: $B_{e x t}=1.0, c_{\|}=1.0, c_{\perp}=2.0$
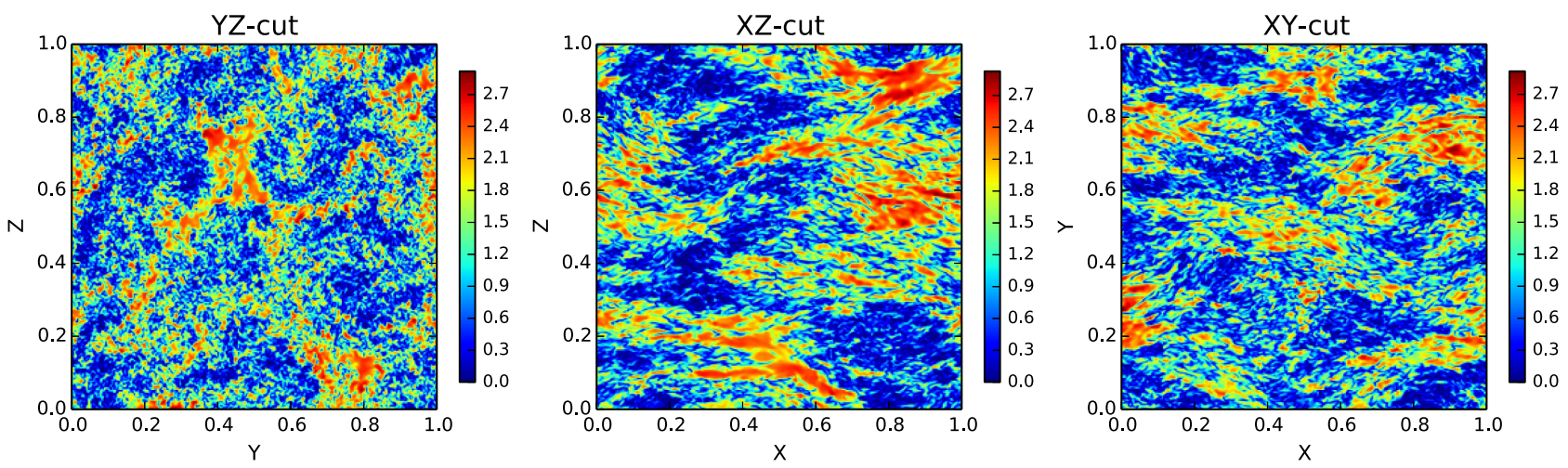

$|\mathrm{B}|$ Cuts - Model: $B_{\text {ext }}=1.0, c_{\|}=1.0, c_{\perp}=0.5$
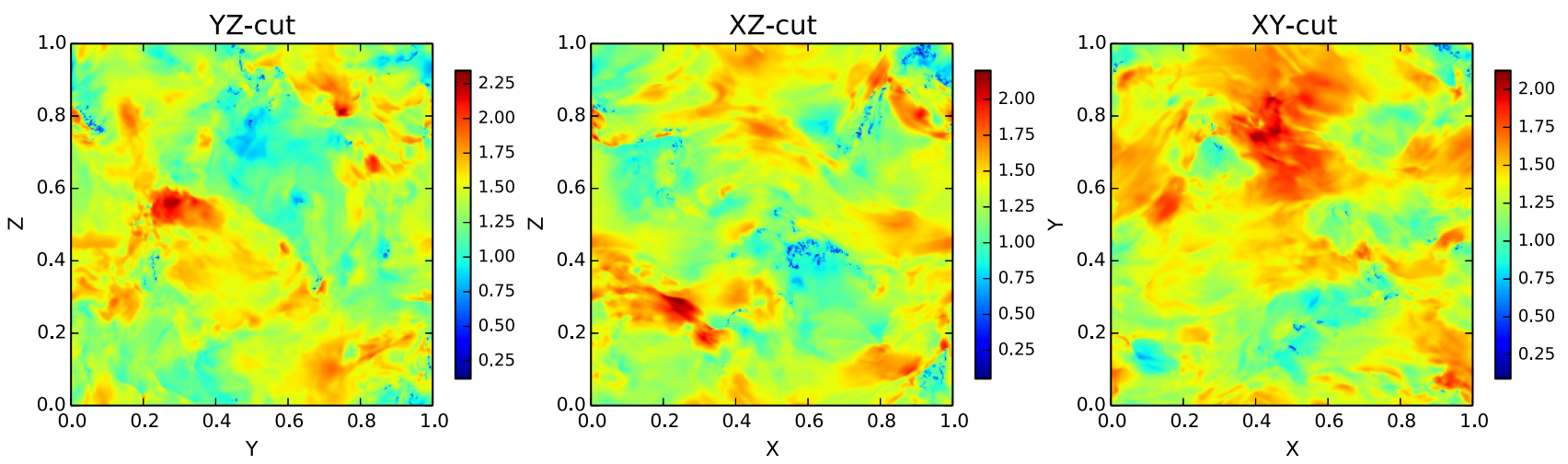

Figure 1. Central slices across the computational domain showing the magnetic field intensity $|\boldsymbol{B}|$ with normal direction along $\hat{x}, \hat{y}$ and $\hat{z}$ (left-hand, middle and right-hand panels, respectively) for collisional Models 1 and 2 (upper row) and collisionless Models 1 and 2 (middle and lower rows, respectively). Each plot has its individual colour scale.

it is enough to reduce the magnetic tension and make the turbulence effectively super-Alfvénic (see the effective Alfvén speed in Table 2). Only in regions where the magnetic field intensity is reduced to values below the threshold (Table 2) the plasma can become unstable, producing the small-scale structures observed in some parts of the map. In general, this model is similar to the collisional MHD case.

The cases corresponding to supersonic and super-Alfvénic regimes are shown in Fig. 2, and the turbulence in these cases is dynamically dominant over the growth of the instabilities. The differences in the magnetic field intensity distribution between the collisional (upper row of Fig. 2) and collisionless MHD models (middle and bottom rows of Fig. 2) are small, being more noticeable by the presence of more structures (at small scales) in Model 3, which becomes mirror unstable for $B<0.34$ (see Table 2). Model 4 , however, is practically stable over its whole volume (the firehose modes can arise only for $B<0.086$ in this case). It can be also observed that in Model 3 the intensities of the magnetic field are smaller than in the corresponding case with isotropic pressure. This is due to the larger effective Alfvén speed, which reduces the magnetic field amplification due to turbulence.

Fig. 3 shows the magnetic field intensity corresponding to the transonic and super-Alfvénic regime. Model 5 can represent qualitatively the conditions in compressed zones in the 
$|\mathrm{B}|$ Cuts - Model: $B_{\text {ext }}=0.1, c_{\text {snd }}=0.1$
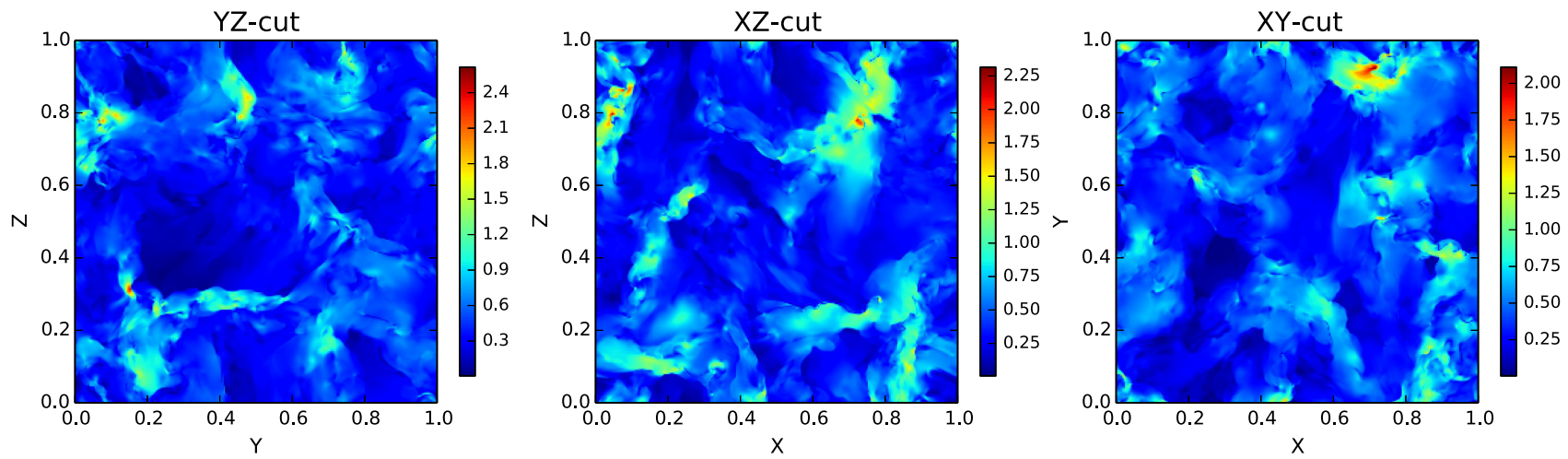

$|\mathrm{B}|$ Cuts - Model: $B_{e x t}=0.1, c_{\|}=0.1, c_{\perp}=0.2$
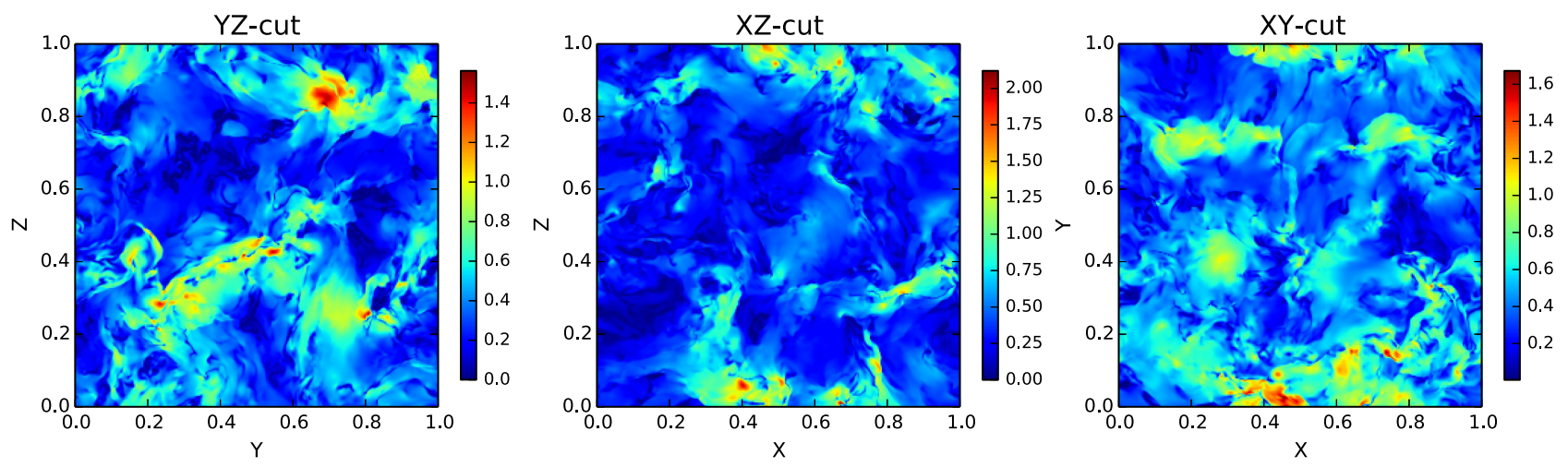

|B| Cuts - Model: $B_{\text {ext }}=0.1, c_{\|}=0.1, c_{\perp}=0.05$
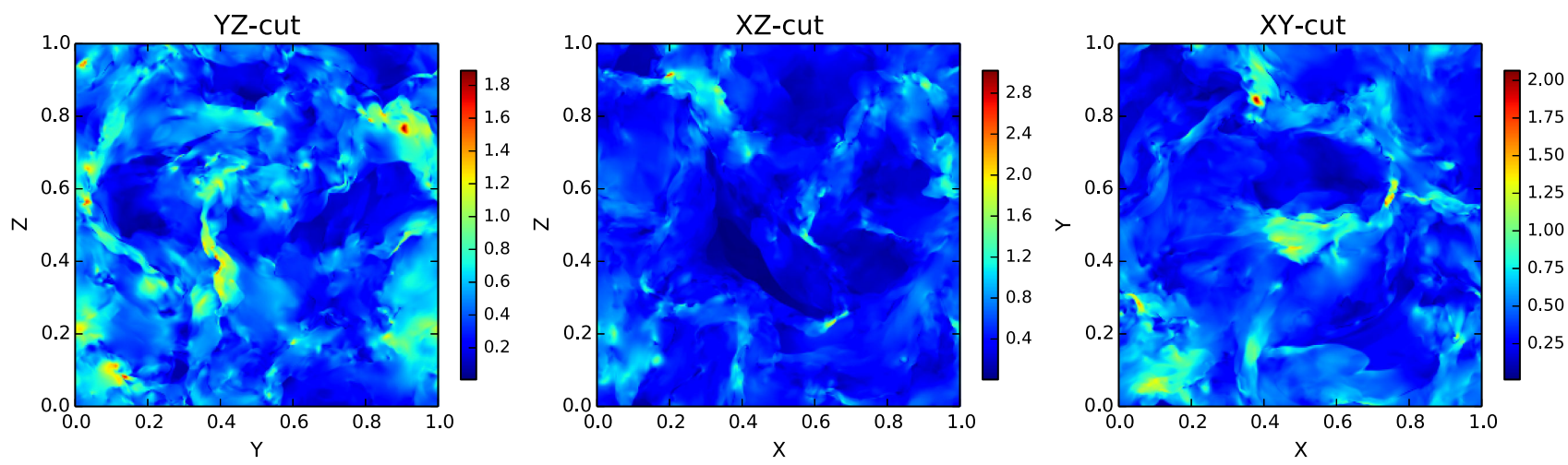

Figure 2. Central slices across the computational domain showing the magnetic field intensity $|\boldsymbol{B}|$ for collisional Models 3 and 4 (upper row), and collisionless Models 3 and 4 (middle and lower rows, respectively).

ICM. The strong firehose instability deforms the magnetic field lines, decreases the anisotropy of fluctuations with respect to the magnetic field lines and produces a very granulated distribution of the magnetic field intensity that is very different from the structure found in the collisional MHD model. The curved magnetic lines tend to slow down and trap the flowing gas in regions of larger magnetic field. ${ }^{4}$ As in Model 1 , the firehose instability can freely

\footnotetext{
${ }^{4}$ We note that the LoS along the initial magnetic field can be easily distinguished from the LoSs along other directions (see the right-hand column). This is, however, just an effect of the different colour scaling. As a matter of fact, in the presence of the firehose instability, the turbulence becomes more
}

grow without being suppressed by the turbulent motions of the gas. It is responsible for the generation of small-scale magnetic field fluctuations and tangling the field lines, which result in an increase of the perpendicular pressure in the local reference frame. Comparing the cases with isotropic and anisotropic pressures, it is seen that in the former the magnetic field intensity is more elongated, and in the latter, several regions of larger magnetic field are formed, while the less intense magnetic field zones are confined to smaller regions.

isotropic because the field lines cannot resist to bending as in super-Alfvénic turbulence. 
|B| Cuts - Model: $B_{e x t}=0.1, c_{\text {snd }}=1.0$
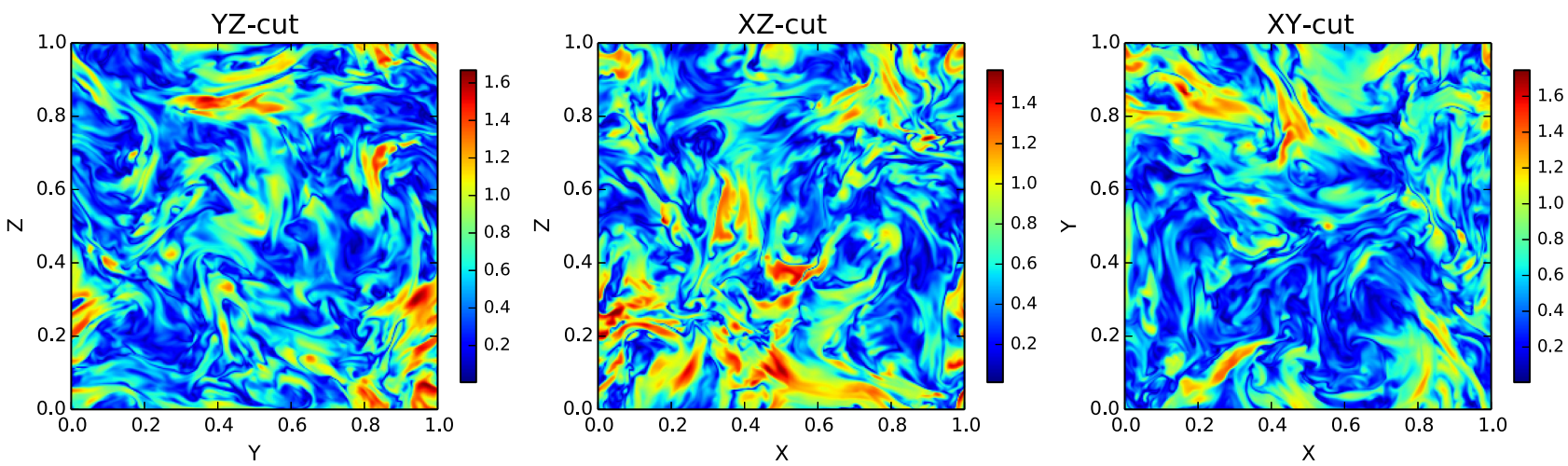

$|\mathrm{B}|$ Cuts - Model: $B_{e x t}=0.1, c_{\|}=1.0, c_{\perp}=0.5$
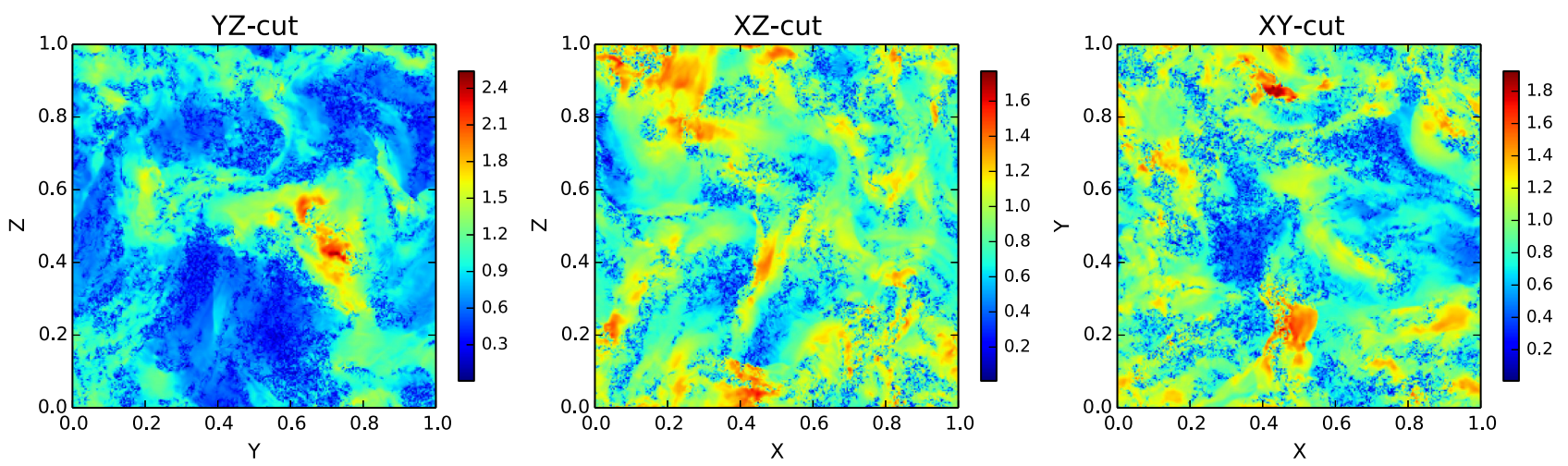

Figure 3. Central slices across the computational domain showing the magnetic field intensity $|\boldsymbol{B}|$ for collisional and collisionless Model 5 (upper and lower rows, respectively).

The magnetic field distribution obtained from Model 6 (corresponding to a supersonic and sub-Alfvénic turbulent regime) is shown in Fig. 4. In this case the collisionless and collisional MHD models are very similar because the sub-Alfvénic turbulence is unable to drive the plasma in Model 6 into the mirror unstable regime given by the condition $B<0.34$ (see Table 2), in which case the thermal pressure anisotropy is dynamically unimportant and does not disturb the turbulent motions.

\subsection{Probability distribution function and power spectra of the magnetic field}

Before analysing the statistics of the FR maps, it is important to revisit the main characteristics of the magnetic field that can be extracted from its PDF and its power spectrum. The first of these two quantities retains information on the distribution of the magnetic field intensity, while the second one on the energy distribution over spatial scales for each turbulent regime. We show the PDFs of the magnetic field intensity in Fig. 5 and the magnetic field spectra in Fig. 6 summarizing the main observed features of these two quantities as an extension of the previous work done by Kowal et al. (2011). Additionally, Table 3 lists the statistical moments (variance, skewness and kurtosis) of the calculated PDFs in both cases.

We should note that the magnetic field intensity PDFs are not expected to follow the Maxwellian distribution. This is from a simple fact, that even though the magnetic field components could in principle follow the Gaussian distribution, we take into account a uniform component $B_{\text {ext }}$ along the X component in our models. The
Maxwellian distribution is expected only if the mean values of the components are zero. The higher statistical moments are used in this section to make qualitative comparison between models, and not to determine the fluctuation randomness or deviation from the Gaussian distribution.

The left-hand panel of Fig. 5 shows the PDF of the magnetic field intensity for the collisional MHD models. It can be seen that for all the regimes studied the PDFs have positive skewness for superAlfvénic turbulence (blue and green lines showing collisional cases corresponding to Models 3-4 and Model 5, respectively) as well as for the sub-Alfvénic turbulence case of Model 6 (red line) in agreement to the values shown in Table 3 . Comparing the values of $|B|$ corresponding to the distribution maxima, or respectively the mean values in Table 3, we see that the distributions are peaked around the values somewhat larger than the initial value of the field $B_{\text {ext }}$ (0.1 for Models 3-5, and 1.0 for Models 1, 2 and 6). This indicates that some sort of magnetic dynamo process takes place in those models, either due to turbulence or kinetic instabilities. For the collisional MHD models it can be seen from Table 3 that the only negative skewness value corresponds to Models 1 and 2 (sub-Alfvénic and transonic regime). Although this value is small, the tendency towards left-hand side can still be observed in the corresponding panel of Fig. 5. On the contrary, the highest value of skewness is observed in super-Alfvénic and supersonic regime with a value reaching over 1.0 and its distribution strongly skewed to the smaller values. Apart from the clear dependence on the initial value of the mean field in the magnetic field intensity PDFs, we distinguish from Fig. 5 that the distributions depend also on the 


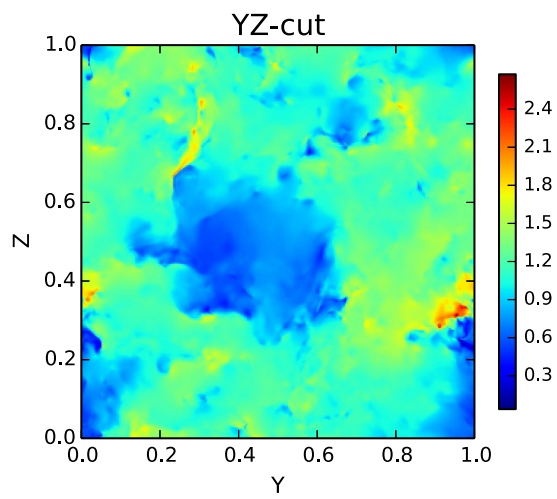

$|\mathrm{B}|$ Cuts - Model: $B_{\text {ext }}=1.0, c_{\text {snd }}=0.1$
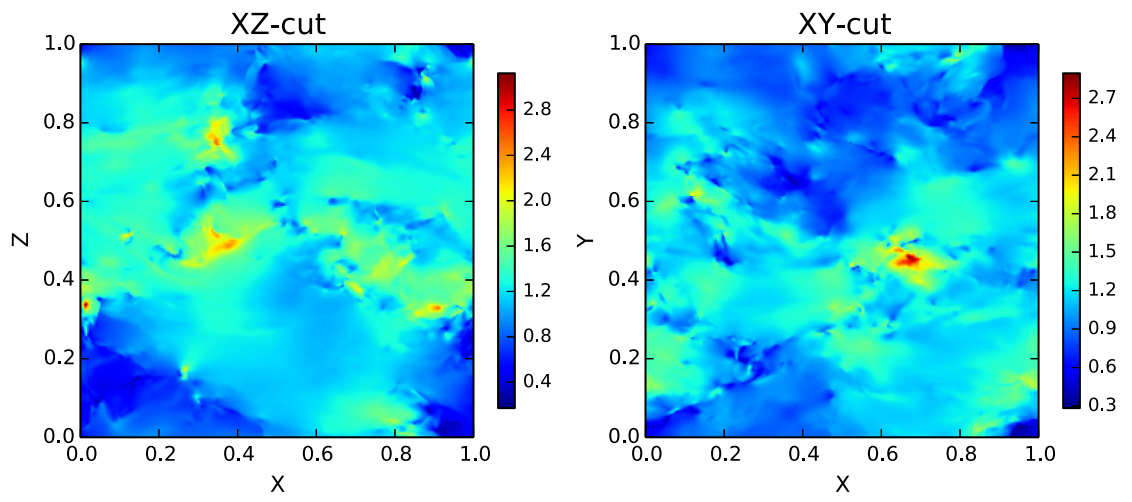

$|\mathrm{B}|$ Cuts - Model: $B_{e x t}=1.0, c_{\|}=0.1, c_{\perp}=0.2$
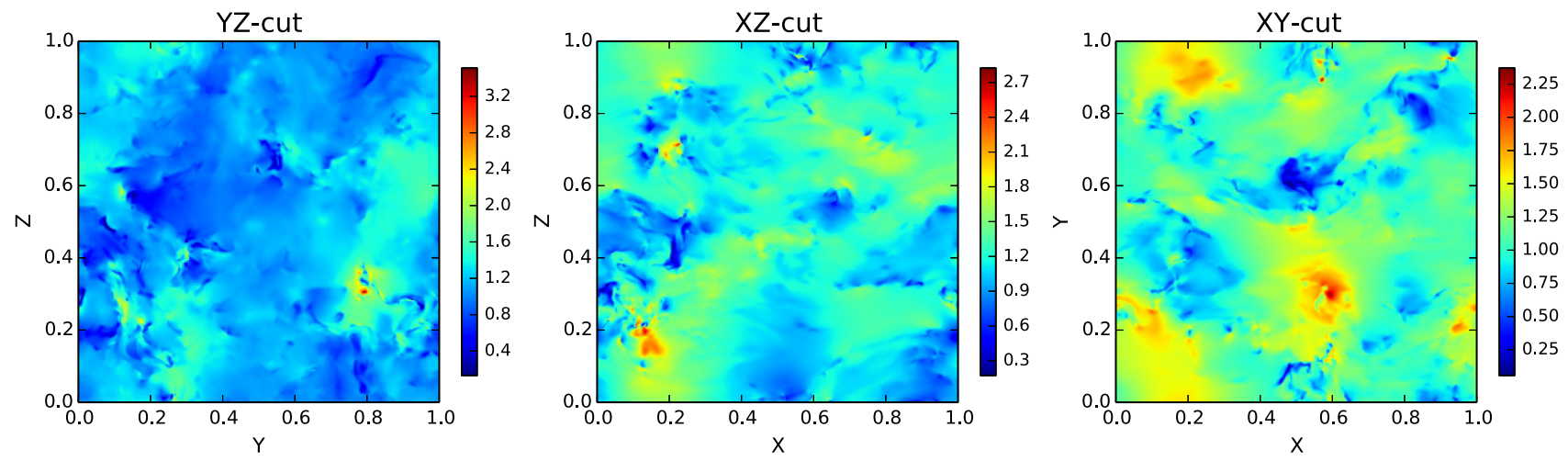

Figure 4. Central slices across the computational domain showing the magnetic field intensity $|\boldsymbol{B}|$ for collisional and collisionless Model 6 (upper and lower rows, respectively).
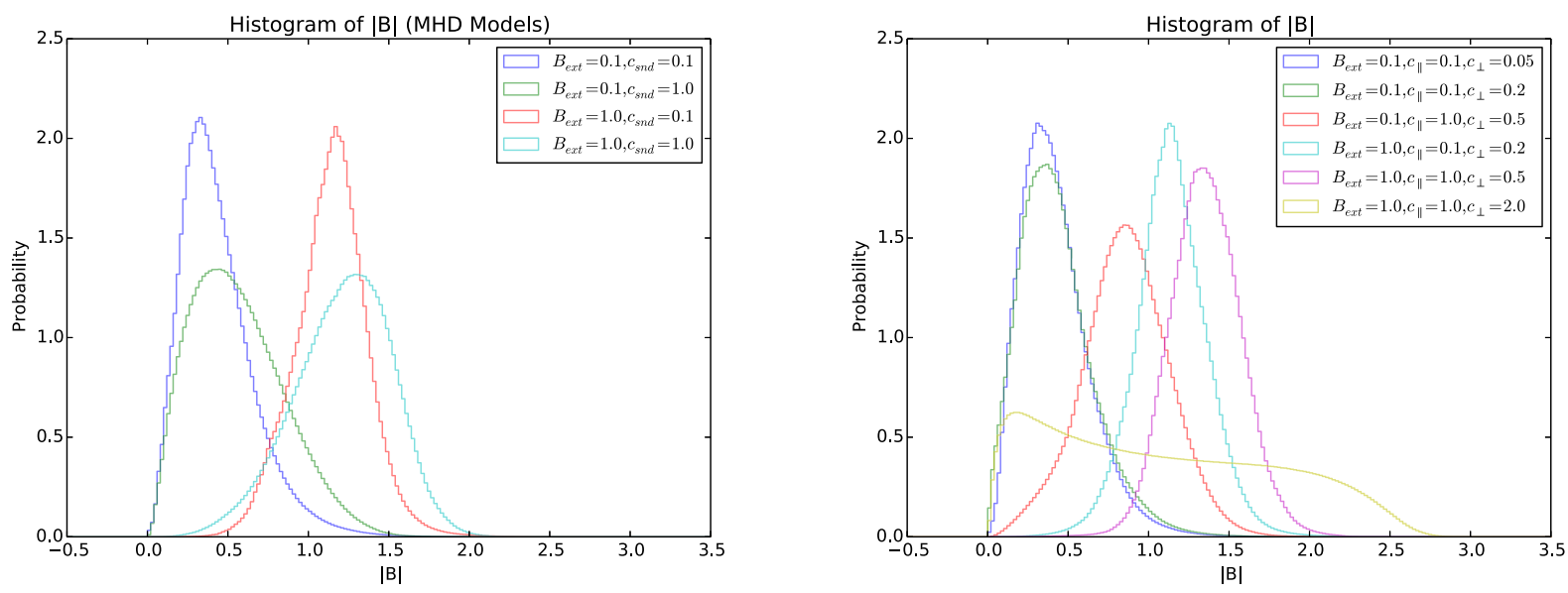

Figure 5. Probability density function (PDF) of the magnetic field intensity for the collisional and collisionless models (left- and right-hand panels, respectively). The models and their parameters are shown in different colours.

sound of speed (or sonic regime). Clearly, for supersonic models the PDFs are more peaked. We notice from both Fig. 5 and Table 3 that the kurtosis of the PDF in the MHD case results either a leptokurtic for the supersonic regime (red and blue lines) or a platikurtic for the subsonic regime (cyan and green lines). This could be interpreted as the presence of infrequent extreme deviations in the values, probably due to the propagation of pressure waves in the leptokurtic case or plasma waves in the platikurtic case (see also Kowal et al. 2011). It is also interesting to note that the variance is in all the cases similar being slightly higher for Models 1 and 2 (cyan line).

The right-hand panel of Fig. 5 shows the PDF for the magnetic field intensity in the collisionless MHD models. The first information one can extract from these plots is the volume fraction of the domain where the firehose and mirror instabilities can develop by observing the volume of the plasma for which the magnetic field intensity is smaller than the threshold values presented in Table 2. 

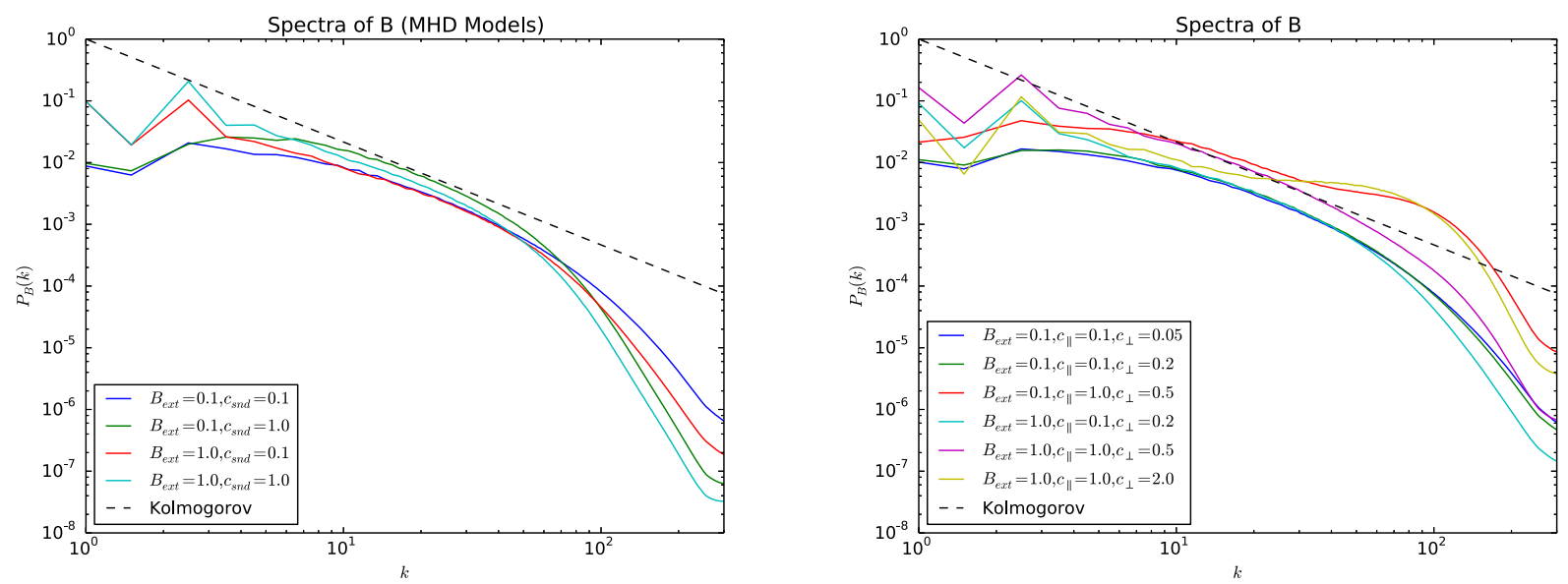

Figure 6. Power spectra of the magnetic field for collisional and collisionless models (left- and right-hand panels, respectively). The models and their parameters are shown in different colours. For comparison, Kolmogorov power spectrum is shown as dashed black line.

Table 3. Statistical moments of the magnetic field intensity $|\boldsymbol{B}|$ for all models from Table 1.

\begin{tabular}{cccrr}
\hline Model & Mean & Std. dev. & Skewness & Kurtosis \\
\hline \multicolumn{5}{c}{ Collisional models } \\
$1-2$ & 1.215 & 0.302 & -0.311 & -0.056 \\
$3-4$ & 0.439 & 0.231 & 1.236 & 2.903 \\
5 & 0.562 & 0.287 & 0.569 & -0.039 \\
6 & 1.143 & 0.226 & 0.167 & 1.419 \\
& \multicolumn{5}{c}{ Collisionless models } \\
1 & 1.091 & 0.706 & 0.306 & -1.052 \\
2 & 1.356 & 0.221 & 0.040 & 0.616 \\
3 & 0.431 & 0.235 & 1.017 & 2.057 \\
4 & 0.421 & 0.217 & 1.128 & 2.682 \\
5 & 0.859 & 0.271 & 0.026 & 0.218 \\
6 & 1.143 & 0.224 & 0.139 & 1.293 \\
\hline
\end{tabular}

We see that only Models 1, 3 and 5 have a no negligible fraction of their volume which is unstable.

Similarly to the standard MHD, two cases corresponding to superAlfvénic turbulence (i.e. Models 3 and 4, green and blue lines, respectively) present positive skewness with similar kurtosis, being both leptokurtic. These models do not demonstrate large deviation from their collisional counterparts. Other models show also positive skewness with much smaller values, however. It is remarkable that according to the values of kurtosis from Table 3, only Model 1 (yellow line) has a negative kurtosis, being clearly platikurtic as shown in Fig. 5. It is particularly noticeable that the conditions prevailing in the ICM (Model 5) result in the most Gaussian-like distribution for the magnetic field intensity with skewness and kurtosis values smaller than for the other models. It is worth to mention that even though the distribution is the closest to Gaussian, the peak is shifted to larger $|B|$ as a result of the firehose instability action leading to larger magnetic field strength values. For the variance there are no noticeable dissimilarities to the MHD cases, with a spreader distribution for Model 1.

The spectral distribution of the magnetic fields in the simulations is quantified by their power spectra shown in Fig. 6. As mentioned in Introduction, many observational works assume a random magnetic field with spectrum following a power law index (Murgia et al. 2004) to link observational data to synthetic FR maps. Through their observational method they find, typically, a power law for the magnetic field spectrum ranging from $-5 / 3$ to $-11 / 3$ when considering different galaxy clusters (see e.g. Murgia et al. 2004; Bonafede et al. 2010a). Previous numerical works based on the collisional MHD description of the ICM (Jones et al. 2011) seem to favour a power law spectrum near $-5 / 3$, thus close to Kolmogorov, justifying the use of this value in the construction of synthetic rotation maps (see e.g. Bonafede et al. 2010b).

The left-hand panel of Fig. 6 shows the power spectrum for the magnetic field in the standard MHD cases. All models present similar spectra with a noticeable inertial range of about one decade in scales and decay at approximately the same lengthscales. The amplitude of the power spectrum within the inertial range is related to the power of the turbulence injected in the simulations. In terms of the slope of the power spectrum all the MHD models seem to present a Kolmogorov-like index close to $-5 / 3$.

In the case of the collisionless MHD models (shown in right-hand panel of Fig. 6) the spectral distribution of the magnetic field may be rather different. Models 2, 3, 4 and 6 (magenta, green, blue and cyan lines, respectively) resembles the MHD cases for all spatial scales with a small departure at larger scales. This reflects the fact that for all these models the instabilities are not significant (or as in the case of Model 3, are not dynamically important). However, for Models 1 and 5 (yellow and red, respectively), which present dynamically important instabilities, there is a noticeable departure from the standard MHD case at small scales. This departure manifests as a bump above the dissipation scales. Models 1 and 5, show that both mirror and firehose instabilities - depending on the turbulent regime - can produce different spatial distributions for the magnetic field lines which may impact the statistics of observables, e.g. FR.

\subsection{Effects of temperature anisotropy on synthetic polarization maps}

As we stated previously, one of the main motivations of our work is to determine the impact of pressure anisotropy, considering a double-isothermal collisionless MHD approximation, on the FR maps. We produced synthetic FR maps considering two different LoSs, along the $\hat{x}$ (parallel to the mean magnetic field direction) and along $\hat{y}$-axis (and equivalently $\hat{z}$-axis, both perpendicular to the mean magnetic field direction) using the simulated magnetic field at the final snapshot of the simulations. These maps are shown in 

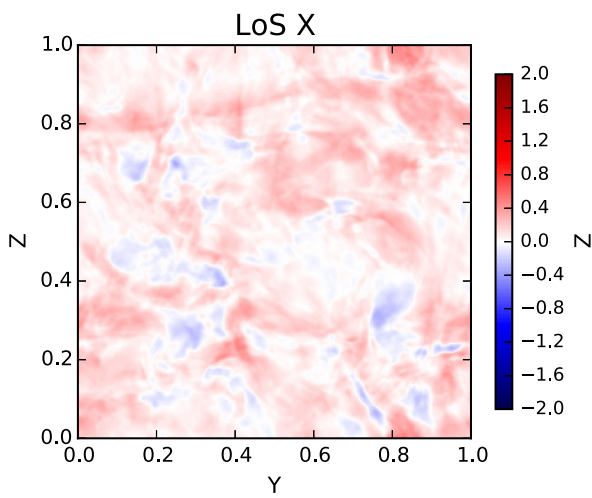

RM Maps - Model: $B_{e x t}=0.1, c_{s n d}=1.0$
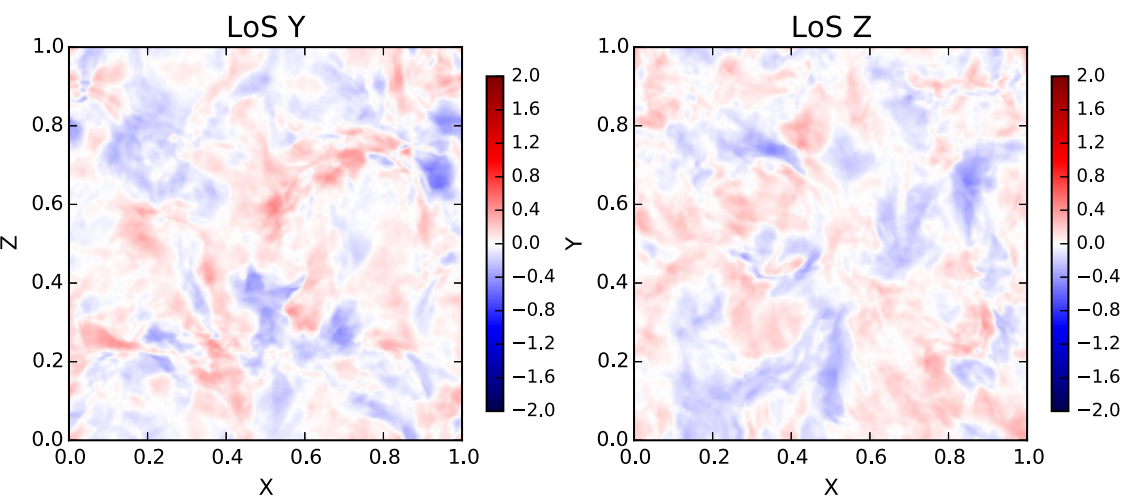

RM Maps - Model: $B_{e x t}=0.1, c_{\|}=1.0, c_{\perp}=0.5$
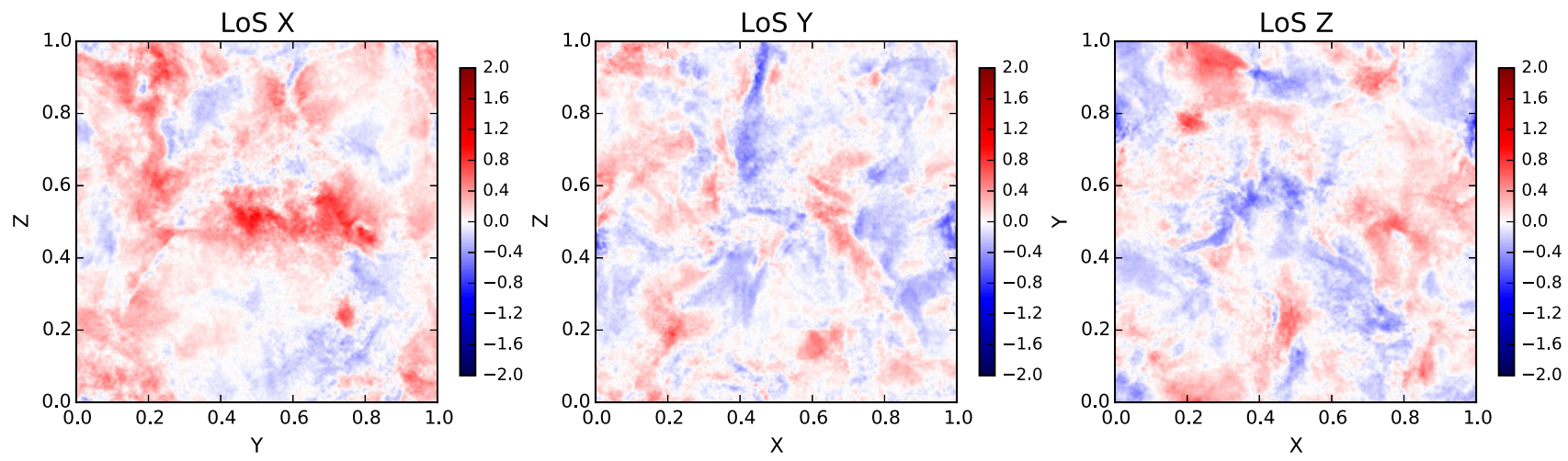

Figure 7. Faraday Rotation maps integrated along the $\hat{x}$-axis, which is parallel to mean field (left-hand column), and perpendicular direction $\hat{y}$ and $\hat{z}$ (middle and right-hand columns, respectively) for collisional and collisionless Model 5 (upper and lower rows, respectively). The maps has the same colour scale.

Figs 8-10. One of the most important things to notice from Figs 8-10 is that the FR maps reveal anisotropies that are not directly evident in the magnetic field intensity (RM is much stronger in the parallel direction comparing to the perpendicular ones, compare left-hand to the middle and right-hand panels of Figs 8-10 and Figs 1-4). This is because, the magnetic field intensity plots show the point contribution at one particular cut of the computational domain, while the FR map takes into account the contribution of magnetic field component parallel to LoS integrated along the computational domain.

Since we are mainly concerned in determining the impact of plasma instabilities on FR maps mimicking the ICM conditions, we start analysing Model 5 and then we compare with the other models. We want to stress not only the effect of such instabilities but also the possibility of having different initial magnetic field configurations leading to different regimes that can rise to similarities in FR maps.

The FR map of Model 5 (Fig. 7), which best resembles the ICM, presents differences between collisional and collisionless approximations. In the collisional case it is not likely to reach a robust conclusion for the initial magnetic field direction due to the resemblance of the maps obtained for the three LoS. This could be because of the super-Alfvénic turbulent regime. The negative and positive domains are parcelled out more or less uniformly in diffuse filaments with no prevalence of any polarity. The turbulence dominates the dynamics of the plasma causing a nearly isotropic distribution of the magnetic field and plasma density which is reflected in the similarity between the maps. For collisionless MHD, on the other hand, the firehose instability gives rise to changes in the magnetic field topology dominating over the fluid motion, but only at small scales. In the LoS parallel to the initial magnetic field, an extended region with higher positive polarity values appears in the central region of the map. The other two maps present similar distributions with thicker filaments alternating positive and negative regions. This small-scale effect gives the appearance of more granulated maps, in comparison to the collisional MHD ones which appear smoother due to the bigger polarization structures of the maps. In terms of the variance of the maps, these results are in agreement with the higher variance values obtained for the collisionless MHD case.

In the case of strong mean field configuration of Model 1 (for collisional and collisionless MHD, see top and middle panels of Fig. 8) the RM distribution for parallel direction is mostly positive and much stronger than along the perpendicular direction to $B_{\text {ext }}$. The map features are clearly different to the ICM case (Model 5). In addition, due to the initial configuration the maps with an LoS parallel to the initial magnetic field show an almost random distribution of positive values. This effect is supported by the smaller values of statistical moments, namely skewness and kurtosis (see Table 4). In the case of Model 2, the map corresponding to parallel LoS differs to the previously discussed cases due to the presence of near zero value regions which reflects the appearance of patterns in this map. Still, this behaviour is not compatible with an ICM-like feature. The variance values are higher in this case than those corresponding to the collisional case and to Model 2. 


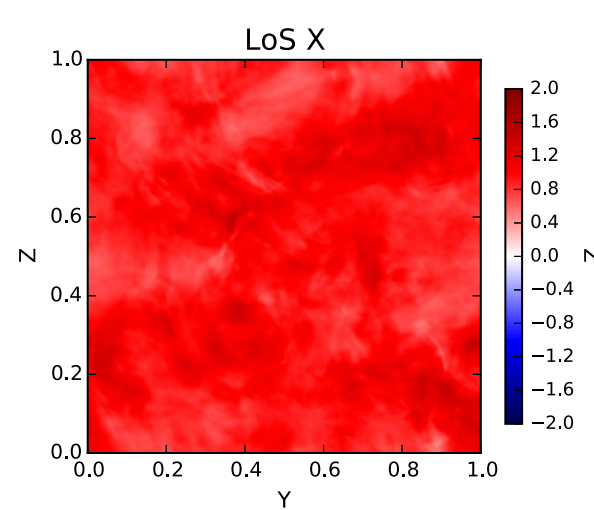

RM Maps - Model: $B_{e x t}=1.0, c_{\text {snd }}=1.0$
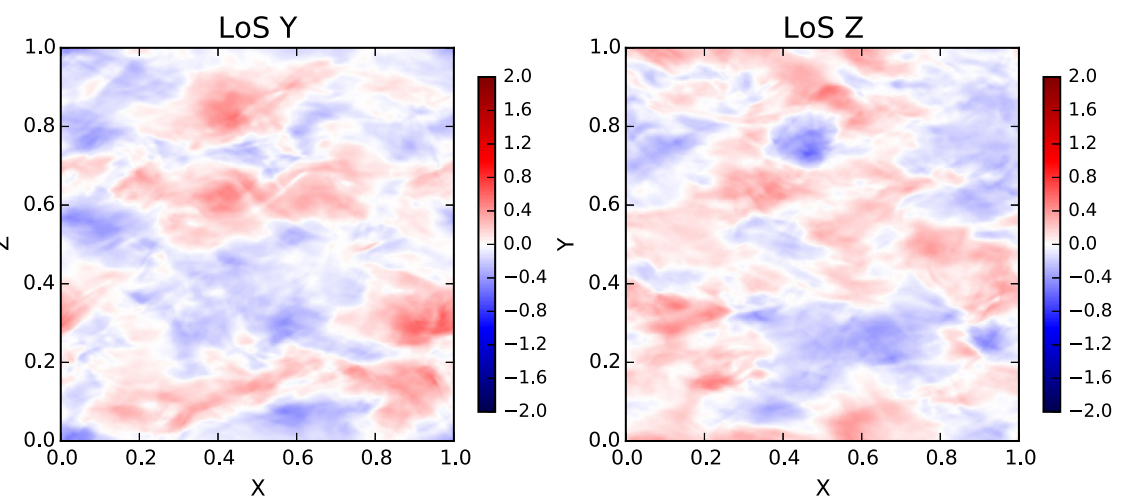

RM Maps - Model: $B_{e x t}=1.0, c_{\|}=1.0, c_{\perp}=2.0$
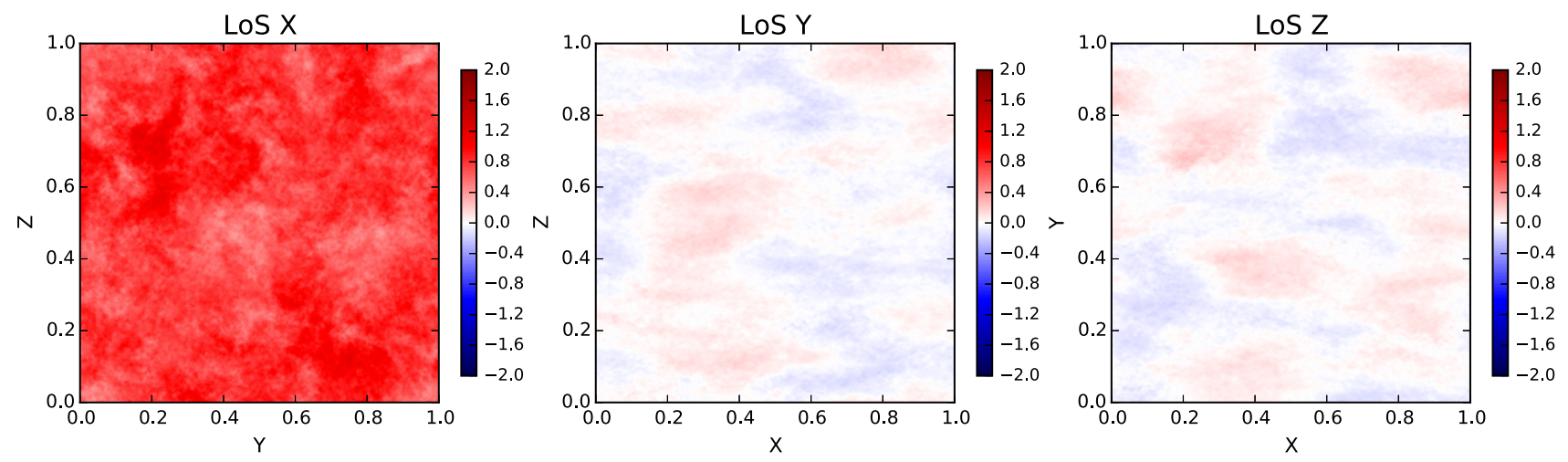

RM Maps - Model: $B_{e x t}=1.0, c_{\|}=1.0, c_{\perp}=0.5$
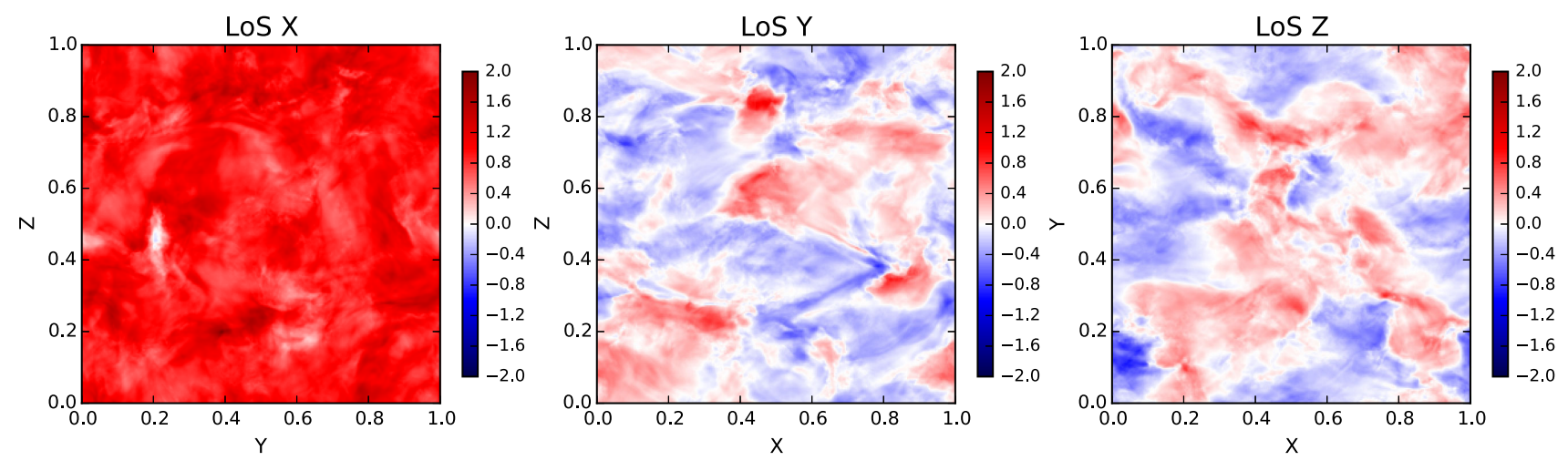

Figure 8. Faraday Rotation maps integrated along the $\hat{x}$-axis, which is parallel to mean field (left-hand column), and perpendicular direction $\hat{y}$ and $\hat{z}$ (middle and right-hand columns, respectively) for collisional Models 1 and 2 (upper row) and collisionless Models 1 and 2 (middle and lower rows, respectively). The maps has the same colour scale.

In the super-Alfvénic turbulence regime, i.e. in Models 3 and 4 (see Fig. 9), the first thing to notice is the fact that in collisional as well as collisionless MHD are not remarkably different. The reason for this effect is the super-Alfvénic turbulence resulting in isotropization of the magnetic fields. When the instabilities are impelled due to the pressure anisotropy (middle and right-hand rows of Fig. 9), the distribution of positive-negative structures on the Faraday maps is diffuse and with the presence of an average polaritydominated region and tiny filaments of opposite polarity. For Model 3 (see bottom row of Fig. 9), the super-Alfvénic turbulence struggles against the mirror modes which propagate nearly perpendicular to the magnetic field. In the case of the stable Model 4, no clear differences between the directions are present. This is supported by similar values for variance being minimal in the perpendicular direction for Model 3 (see Table 4 and middle panel of Fig. 9). Taking into consideration these results, which depart from those obtained for ICM, it would be unexpected to find Faraday maps with such characteristics in environments like the ICM, even though these three models represent cases of super-Alfvénic turbulent regime.

Finally, Fig. 10 shows the FR maps obtained for turbulence in supersonic regime. In consistency with the analysis of Model 6 in 

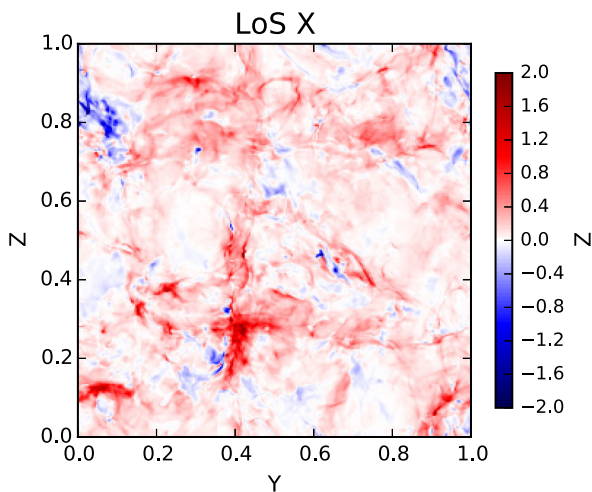

RM Maps - Model: $B_{e x t}=0.1, c_{\text {snd }}=0.1$
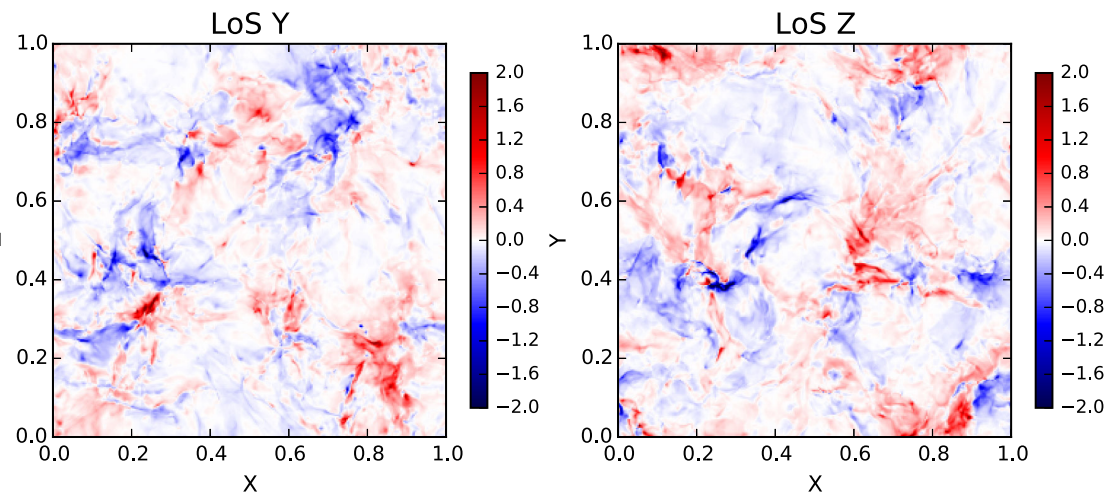

RM Maps - Model: $B_{e x t}=0.1, c_{\|}=0.1, c_{\perp}=0.2$
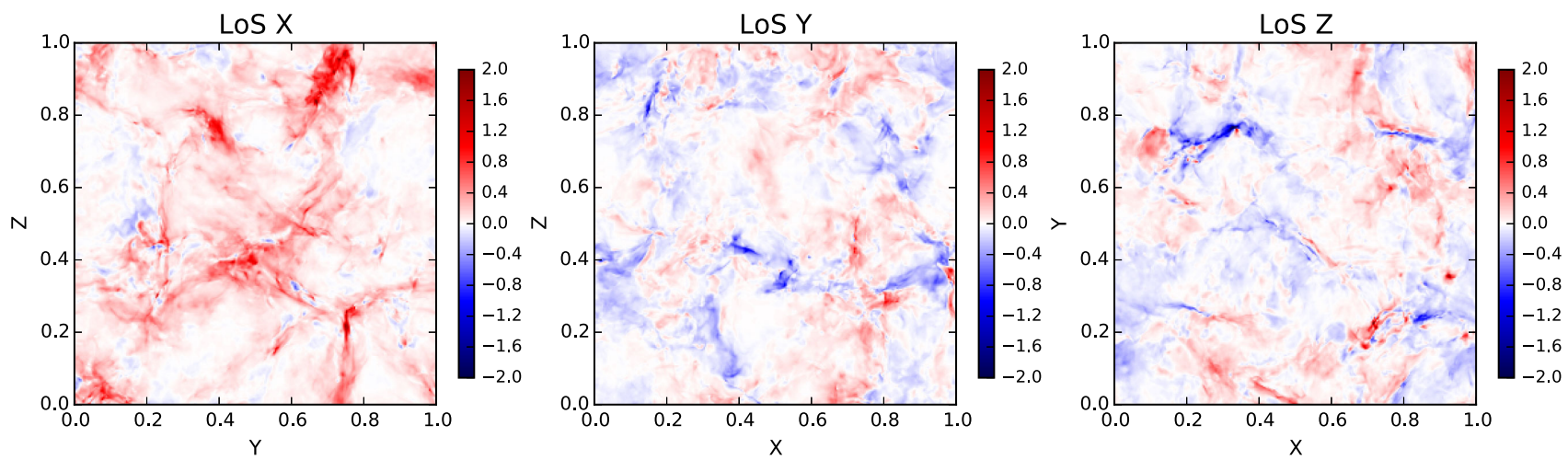

RM Maps - Model: $B_{e x t}=0.1, c_{\|}=0.1, c_{\perp}=0.05$
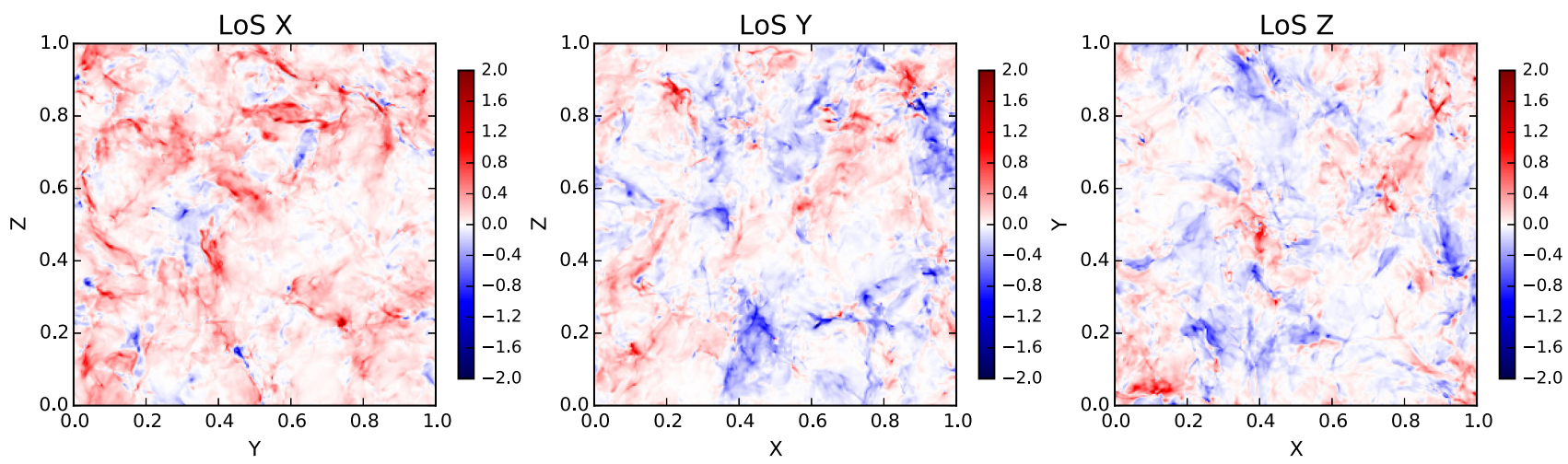

Figure 9. Faraday Rotation maps integrated along the $\hat{x}$-axis, which is parallel to mean field (left-hand column), and perpendicular direction $\hat{y}$ and $\hat{z}$ (middle and right-hand columns, respectively) for collisional Models 3 and 4 (upper row) and collisionless Models 3 and 4 (middle and lower rows, respectively). The maps has the same colour scale.

the previous section, we see that there is no significant differences between the collisional and collisionless cases, only that in the $\hat{x}$ LoS the values of the Faraday maps are larger and near randomly distributed. This is because most of the domain in the collisionless model is stable as supersonic turbulence suppresses the instabilities. The filamentary distribution in the maps corresponding to the LoS in the perpendicular directions are clearly well defined. The spread of the filaments is in agreement with the variance values of Table 4 being the highest among all models.

\subsection{Probability distribution function and power spectra of the Faraday rotation maps}

As mentioned in Introduction, observational works are compelled to assume a Gaussian distribution for the magnetic field component along the LoS used to compute synthetic FR. This is the simplest assumption one can adopt. Here we investigate synthetic RM maps obtained from self-consistent magnetic field distributions from three-dimensional numerical simulations of turbulence which allow for direct derivation of their statistical properties, such as the 
RM Maps - Model: $B_{\text {ext }}=1.0, c_{\text {snd }}=0.1$
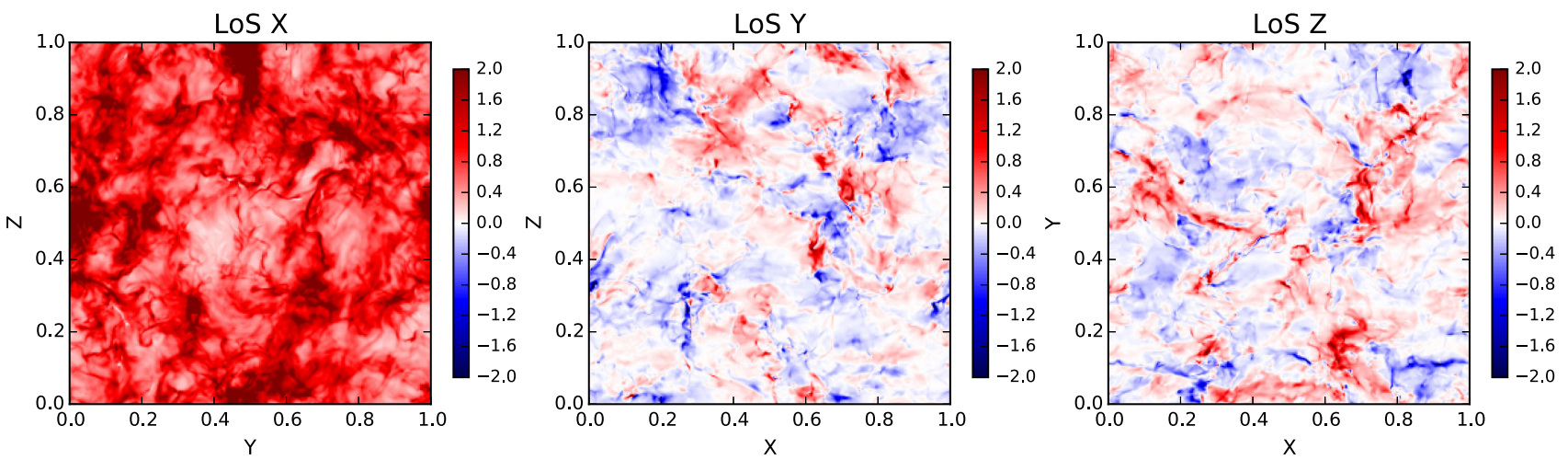

RM Maps - Model: $B_{e x t}=1.0, c_{\|}=0.1, c_{\perp}=0.2$
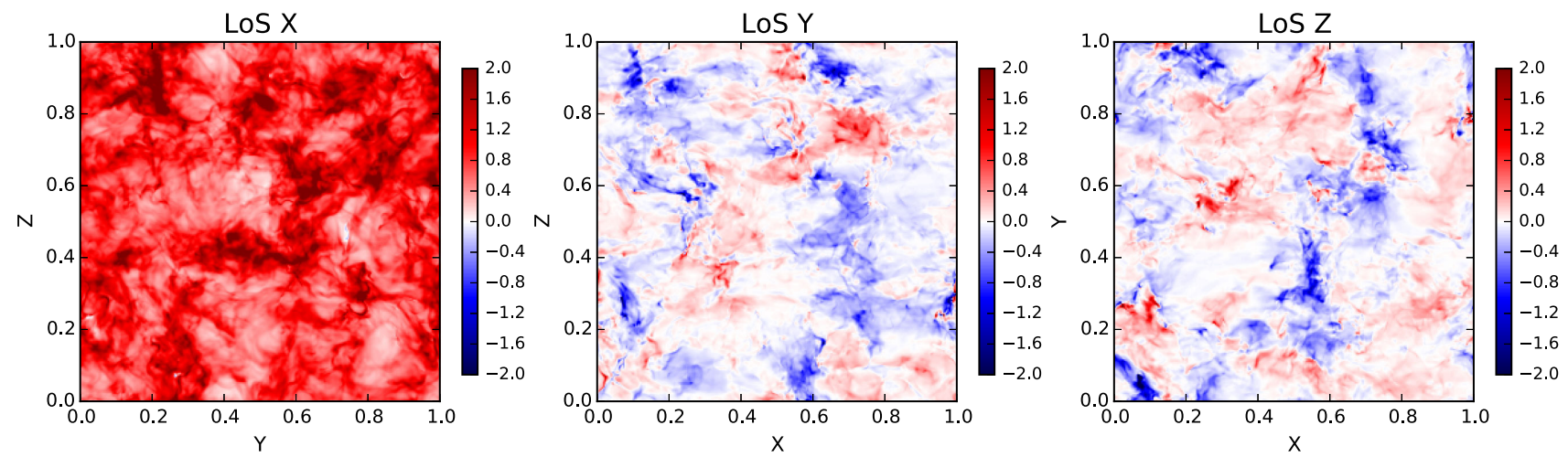

Figure 10. Faraday Rotation maps integrated along the $\hat{x}$-axis, which is parallel to mean field (left-hand column), and perpendicular direction $\hat{y}$ and $\hat{z}$ (middle and right-hand columns, respectively) for collisional and collisionless Model 6 (upper and lower rows, respectively). The maps has the same colour scale.

Table 4. Statistical moments for the RM maps integrated along the parallel $(\|)$ and perpendicular $(\perp)$ directions to the mean field for all models from Table 1.

\begin{tabular}{|c|c|c|c|c|c|}
\hline Model & LoS & Mean & Std. dev. & Skewness & Kurtosis \\
\hline \multicolumn{6}{|c|}{ Collisional models } \\
\hline \multirow[t]{2}{*}{$1-2$} & $\|$ & 0.944 & 0.150 & 0.323 & -0.248 \\
\hline & $\perp$ & 0.006 & 0.160 & 0.386 & 0.342 \\
\hline \multirow[t]{2}{*}{$3-4$} & $\|$ & 0.116 & 0.201 & 0.976 & 14.000 \\
\hline & $\perp$ & -0.001 & 0.198 & 0.451 & 17.063 \\
\hline \multirow[t]{2}{*}{5} & $\|$ & 0.091 & 0.104 & 0.202 & 0.571 \\
\hline & $\perp$ & 0.005 & 0.119 & -0.231 & 1.238 \\
\hline \multirow[t]{2}{*}{6} & $\|$ & 0.971 & 0.559 & 2.050 & 7.802 \\
\hline & $\perp$ & -0.006 & 0.253 & 0.180 & 9.522 \\
\hline \multicolumn{6}{|c|}{ Collisionless models } \\
\hline \multirow[t]{2}{*}{1} & $\|$ & 0.777 & 0.130 & 0.207 & 0.075 \\
\hline & $\perp$ & 0.005 & 0.058 & 0.163 & -0.356 \\
\hline \multirow[t]{2}{*}{2} & $\|$ & 0.942 & 0.193 & 0.083 & 1.101 \\
\hline & $\perp$ & -0.019 & 0.242 & 0.466 & 0.336 \\
\hline \multirow[t]{2}{*}{3} & $\|$ & 0.117 & 0.169 & 2.359 & 10.580 \\
\hline & $\perp$ & -0.006 & 0.139 & -0.355 & 5.743 \\
\hline \multirow[t]{2}{*}{4} & $\|$ & 0.112 & 0.175 & 1.218 & 10.100 \\
\hline & $\perp$ & -0.009 & 0.185 & -0.677 & 15.303 \\
\hline \multirow[t]{2}{*}{5} & $\|$ & 0.105 & 0.185 & 0.838 & 1.195 \\
\hline & $\perp$ & -0.004 & 0.156 & 0.005 & 1.075 \\
\hline \multirow[t]{2}{*}{6} & $\|$ & 0.897 & 0.433 & 1.287 & 2.868 \\
\hline & $\perp$ & -0.036 & 0.232 & -0.682 & 7.288 \\
\hline
\end{tabular}

probability distribution function and the power spectrum. The PDF of RM for each model can be seen in Fig. 11 (upper panels for collisional and lower panels for collisionless models) and the statistical moments of the RM distributions are given in Table 4.

The synthetic RM maps are obtained from our cubes by integrating the product of density and magnetic field component parallel to the integration direction, i.e.:

$\mathrm{RM}=\frac{1}{L} \int_{0}^{L} \mathrm{~d} l \rho(l) B_{n}(l)$,

where $L$ is the size of the simulated cube, $l$ is the position along the integrated direction (our LoS) and $B_{n}$ is the magnetic field component parallel to the integration direction (or normal to the plane of map). With this definition, the mean value of RM over the plane of sky is given by

$\overline{\mathrm{RM}}=\rho_{0} \bar{B}_{n}+\frac{1}{L} \overline{\int_{0}^{L} \mathrm{~d} l \delta \rho(l) \delta B_{n}(l),}$

where $\bar{B}_{n}$ is the mean of the magnetic field component $B_{n}(l), \rho_{0}$ is the average density (equal to unity in all our simulations), $\delta B_{n}(l)=$ $B_{n}(l)-\bar{B}_{n}$ is the fluctuating part of magnetic field components and the bar denotes the average over the plane of sky. The last term in equation (12) is zero if both the density and magnetic field fluctuations have normal distributions. In such case, $\overline{\mathrm{RM}}=\rho_{0} \bar{B}_{n}$, and, e.g. if the integration is done along the $\hat{x}$ direction, $\overline{\mathrm{RM}}=B_{\text {ext }}$, or if the integration is done along the $\hat{y}$ or $\hat{z}$ directions, $\overline{\mathrm{RM}}=0$. In the next paragraphs we will see, that the mean values of RM deviate 

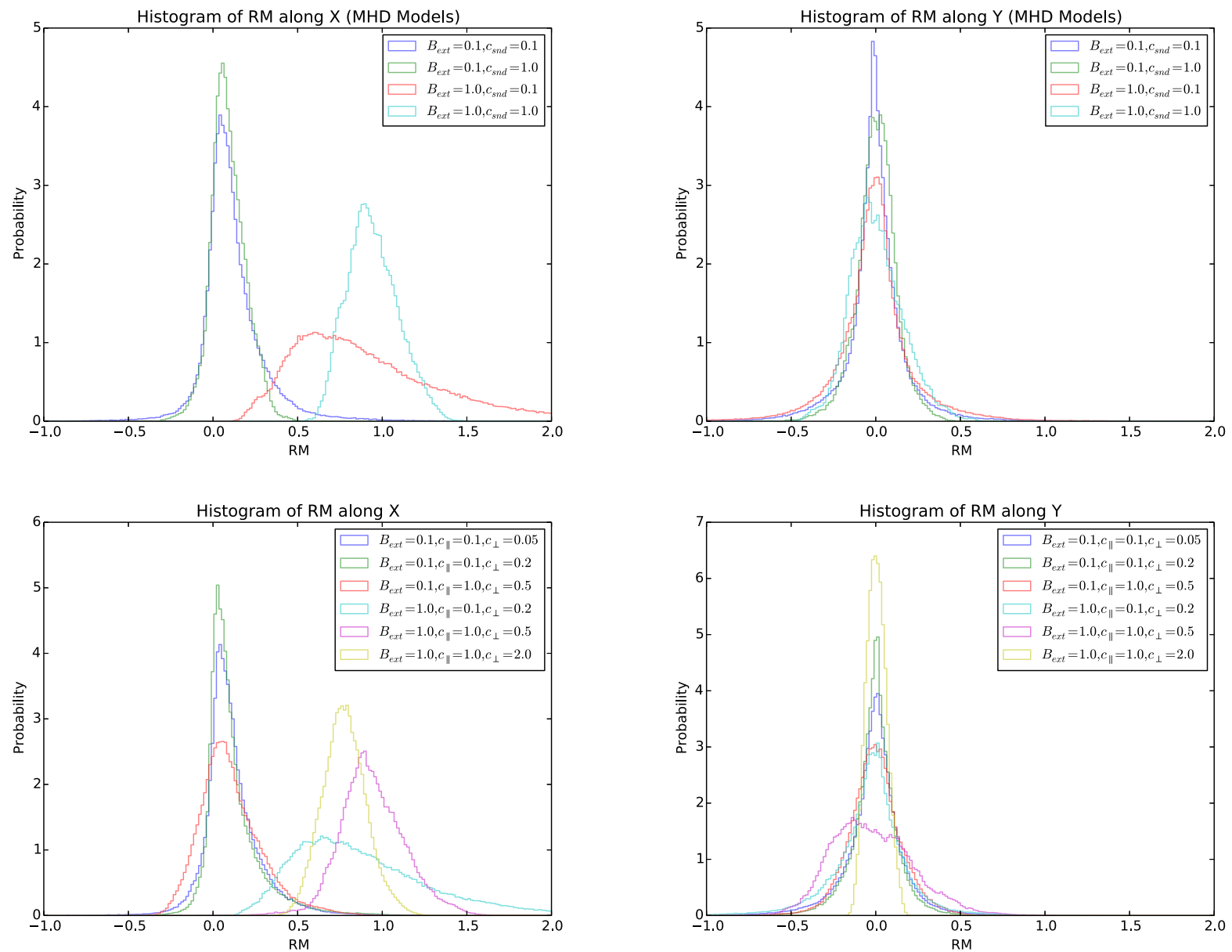

Figure 11. Probability distribution function of the RM maps corresponding to the collisional and collisionless models (upper and lower rows, respectively). Left- and right-hand columns show the RM integration along the parallel and perpendicular direction to $B_{\text {ext }}$, respectively.

from these values, indicating that the distributions of $\delta \rho$ and $\delta B_{n}$ are not described by Gaussian distributions. The spread (or width) of the RM distribution $\sigma_{\mathrm{RM}}$ can be approximately given by

$\sigma_{\mathrm{RM}}^{2} \sim \delta B_{f}^{2}+\bar{B}_{n} \delta \rho_{f}^{2}$,

where $\delta B_{f}^{2}$ and $\delta \rho_{f}^{2}$ are the power of the magnetic and density fields at the scales of the energy injection.

Analysing the mean RM for collisional models in Table 4, we see that for the LoS perpendicular to the mean magnetic field, $\overline{\mathrm{RM}} \approx 0$ (the difference is always smaller than 0.01 ), while for the parallel LoS, it is smaller than $B_{\text {ext }}$ for Models $1,2,5$ and $6(6,9$ and 3 per cent smaller, respectively) and larger for Models 1 and 2 (16 per cent larger). In the parallel RM maps of the collisional models (top left panel of Fig. 11), subsonic models (green and cyan lines) present normal-like distribution of RM. Two subsonic models demonstrate deviation from the normal distribution in the high-value tail (Model 6 shows extremely elongated tail justifying the large value of skewness in Table 4). This is most probably due to the lognormal distribution of the density in the highly supersonic regime (see Kowal et al. 2007). Both super-Alfvénic models do not differ much, only the less peaked form of the curve characterizes Model 5, due to the mentioned lognormal distribution of density. For the perpendicular LoS, we see in the right top panel of Fig. 11 that the width of the distribution of the RM measurements are all similar (see the standard deviation column in Table 4). For the parallel LoS, models with low $B_{\text {ext }}$ show similar spread, even though Models 3 and
4 (blue line) is supersonic, for which the contribution of the density fluctuations is of minor importance as it is multiplied by small $B_{\text {ext }}$. However, when comparing models with larger $B_{\text {ext }}$ (Models 1, 2 and 6, cyan and red lines in Fig. 11, respectively), we clearly see that the spread of the RM distribution of Model 6 is much larger due to the supersonic turbulence (also, see the comparison between both density power spectra in Kowal et al. 2011).

Now, let us focus on how the collisionless models deviate from their collisional counterparts. First, we notice from Table 4, that Model 1 is much lower mean value of RM than its collisional counterpart. This model has RM distribution relatively symmetric, however, strongly shifted to the lower values (see yellow line in bottom left panel). This must be the effect of strong mirror instability operating here. The mean RM for Models 2-5 do not differ much comparing with their collisional counterparts, including Model 5 which best describes the ICM. Model 6 demonstrates lowered mean value of RM, although its distribution is similar to its collisional counterpart (see cyan line in the left lower panel and red line in the left upper panel, for collisionless and collisional cases, respectively). For perpendicular LoS (the right bottom panel in Fig. 11), the values of $\overline{\mathrm{RM}}$ are around zero (see also Table 4), similarly to the collisional case. Only Models 1 and 6 show mean values slightly higher than others in this direction. The width of the RM distributions, however, for Model 1 (yellow line) is smaller, while for Model 2 (magenta line) is larger. Other models do not show significant differences with respect to their collisional counterparts, even 

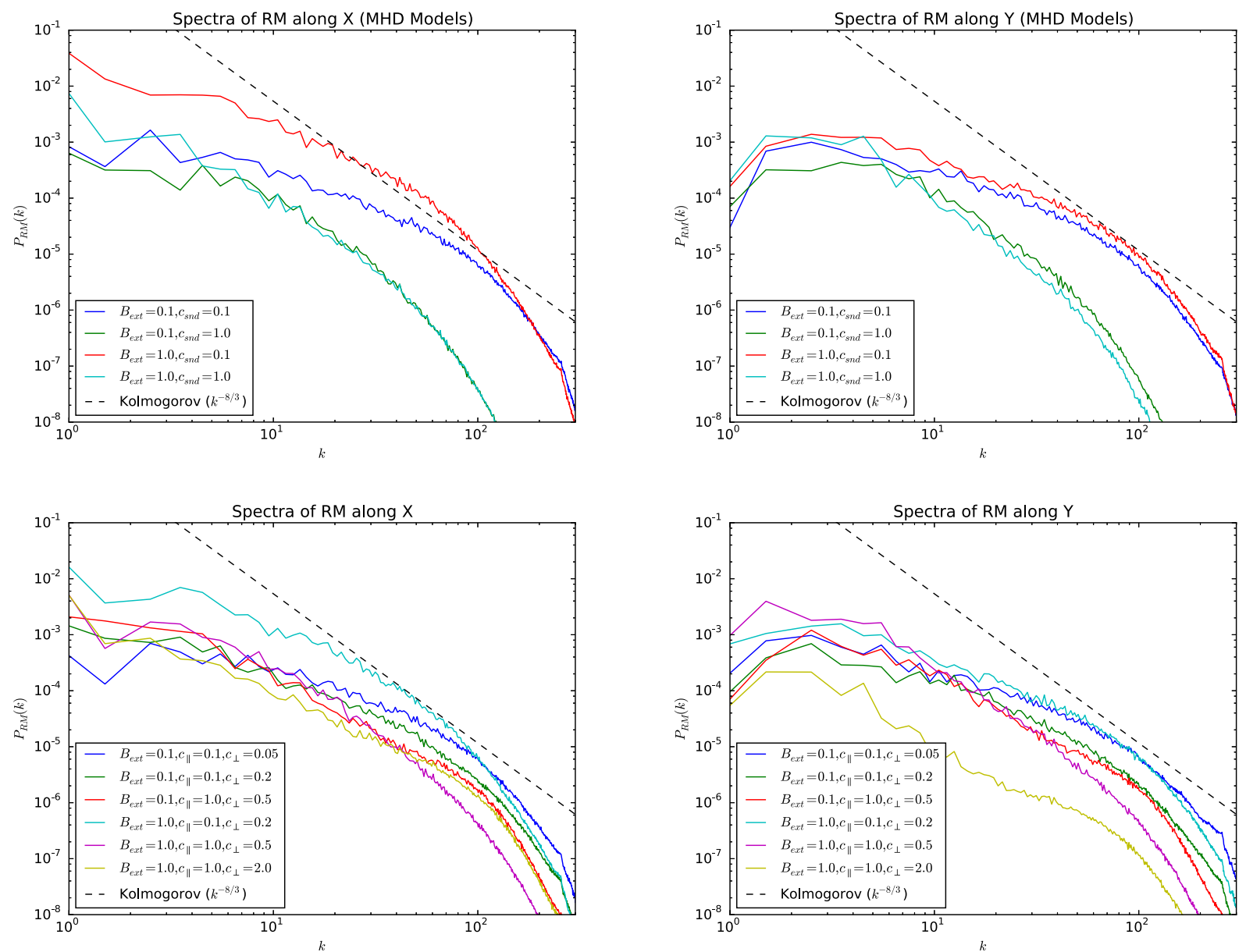

Figure 12. Power spectra of RM for collisional and collisionless models (upper and lower panels, respectively). Spectra for RM integrated along the parallel and perpendicular direction to $B_{\text {ext }}$ are shown in the left- and right-hand columns, respectively. The 2D Kolmogorov power spectrum $\left(k^{-8 / 3}\right)$ is shown in dashed black line for comparison.

Model 5 representing the ICM. With regard to the width $\sigma_{\mathrm{RM}}$ of the distribution, we observe its reduction for Models 1 and 3 (yellow and green lines, respectively) while it increases for Models 2 and 5 (magenta and red lines). It is easy to understand these differences in terms of the changes in the effective Alfvén speed (which increases for Models 1 and 3 and decreases for Models 2 and 5).

For perpendicular LoS, it can be seen in Fig. 11 that all curves, except collisionless Model 2, lie almost in the same region as the collisional models. All plots being similarly symmetric and leptokurtic. The RM distribution of collisionless Model 2 is strongly deformed.

Now, let us evaluate the higher order statistical moments of the distributions. For parallel LoS, all the models in both collisional and collisionless cases lead to positive skewness values with a minimum value corresponding to collisionless Model 2, which is much smaller than in its collisional counterpart (see Table 4). Model 5, representing the ICM conditions, has relatively large value of skewness, much higher than the corresponding collisional model. Similarly, collisionless Models 3 and 4 have larger skewness when comparing to their collisional counterpart. In Model 6, the skewness of distribution decreases after including pressure anisotropy.

Looking at the kurtosis column in Table 4, we see that for the perpendicular maps, most of the distributions are strongly peaked (kurtosis $>1.0$ ). The Model 1 is slightly platykurtic, while Model 2 is slightly leptokurtic. The ICM Model 5 has kurtosis 1.1 in the direction perpendicular to mean field, which is not much different for the corresponding collisional case. The biggest difference in distribution between collisional and collisionless cases in the perpendicular map is observed for Model 2, which is much more spread comparing to its collisional counterpart.

The power spectra of RM obtained for each model is shown in Fig. 12. For collisional models (upper panels), the remarkable is the distinction between both the transonic (Models 1, 2 and 5) and supersonic regimes (Models 3, 4 and 6) (cyan-green versus blue-red lines, respectively) for both directions.

In the limit of anisotropic turbulence and neglecting any magnetic-density phase correlation, the RM power spectrum can be related to the density and magnetic power spectra in the following way:

$\mathcal{P}_{\mathrm{RM}}(k) \propto\left(\rho_{0}^{2} \frac{P_{B}(k)}{k}+\bar{B}_{n}^{2} \frac{P_{\rho}(k)}{k}\right)$.

In the case of the perpendicular $\operatorname{LoS}\left(\bar{B}_{n}=0\right)$, the estimative above states that only the magnetic power spectrum influences $\mathcal{P}_{\mathrm{RM}}(k)$. In the transonic cases (green and cyan lines of Fig. 12, upper panel), the slopes seem to be compatible with $k^{-8 / 3}$ (as it is expected if $P_{B}(k) \sim k^{-5 / 3}$ ). In the supersonic cases, we have also the contribution of magnetic fluctuations coming from the magnetosonic modes. As the magnetic spectrum of the fast modes is flatter ( $\sim k^{-3 / 2}$ according to Cho \& Lazarian 2002), we could expect the 
magnetic spectrum to be flatter. Indeed, we see such feature in the magnetic power spectrum of Fig. 6, but it is not enough to explain the difference seen in the RM spectra (which satisfies $\sim k^{-1}$ ). Therefore, such difference must comes from the contribution of the combined density and magnetic fluctuations from the compressible modes in the smaller scales (larger $k$ values). Comparing with the parallel LoS case, the subsonic models (cyan and green lines) have slopes similar to the perpendicular LoS. In these cases, the density fluctuations are small and do not affect the $\mathcal{P}_{\mathrm{RM}}(k)$. Also for the supersonic and super-Alfvénic Models 3 and 4 (blue line), parallel LoS does not differ from the perpendicular LoS case. This is due to the fact that the contribution from the density fluctuations are weighted by the $\bar{B}_{n}$, which is small $\left(B_{\mathrm{ext}}=0.1\right)$ in this case. Only the sub-Alfvénic, supersonic Model 6 (red line) differs in the parallel LoS. Here, the contribution from the density fluctuations seems to increase the power in the large scales, although the slope seems steeper than in the perpendicular LoS.

Now, let us examine the differences in the $\mathcal{P}_{\mathrm{RM}}$ for the collisionless models show in the lower row of Fig. 12. The ICM representative Model 5 (red line) looks similar to its collisional counterpart in large scales, however, in the small-scale range the fluctuations of $\mathrm{RM}$ are stronger. The similar situation is seen in the perpendicular LoS (the bottom right panel). It is due to the enhanced magnetic fluctuations originating from the firehose instability. The transonic Model 2 (violet) and Models 4 and 6 are also similar to its collisional counterpart in both LoSs. For the perpendicular LoS, Model 1 (yellow line) presents less power in most of the scales, compared to the collisional case. Also, at the large scales, the power law seems to be deeper. The decrease (increase) of magnetic power in the large (small) scales (see Fig. 6) cannot explain such differences, so it must be a combined effect of the density-magnetic field fluctuations (which have negative correlation for mirror modes). For the parallel LoS, however, we can observe an increase of the power only at the small scales. It can be due to the increase of the density power at the same scales (see Kowal et al. 2011).

\subsection{Autocorrelation functions of Faraday rotation maps}

We compute the isotropic autocorrelation function of the simulated FR maps as follows:

$$
A(l)=\langle\operatorname{RM}(\boldsymbol{r}) \operatorname{RM}(\boldsymbol{r}+\boldsymbol{l})\rangle,
$$

where $\langle\cdots\rangle$ stands for the average taken over all positions $\boldsymbol{r}=(x, y)$ in the FR maps and directions of the shift $l=|l|$. It can be related to the power spectrum of $\mathrm{RM}, \mathcal{P}_{\mathrm{RM}}(k)$, in the plane by

$A(l) \propto \int_{0}^{\infty} \mathrm{d} k \mathcal{P}_{\mathrm{RM}}(k) \cos (k l)$,

where $k$ is the wavevector (see a more detailed analysis in Enßlin $\&$ Vogt 2003). The use of this function enables us to quantify the statistical properties of the magnetic field structure. In particular, it is possible to extract an average correlation length, which gives an idea of the extension of patches in the structure of the field. This quantity can be observed in Fig. 13, which shows the collisional cases in the upper panels and the collisionless ones in the lower panels.

In sub-Alfvénic supersonic collisional model (red line in top panels) the correlation length along the perpendicular direction is much shorter than along the parallel one, indicating strong anisotropy of the RM structures. In all remaining collisional models, the correlation lengths along both directions are comparable, demonstrating very small degree of anisotropy of RM. We can recognize, that for a given sound speed, the parallel correlation length increases with the strength of mean field. This is not observed in perpendicular direction for supersonic models, for which the correlation length are almost insensitive to $B_{\text {ext }}$ (red and blue lines in top right panel).

For collisionless models, a different trend is observed (see lower panel in Fig. 13). Model 5 (red line), which best resembles ICM, shows strong anisotropy with parallel correlation length $l_{\|} \sim 39$ and perpendicular one $l_{\perp} \sim 22$, on the contrary to the corresponding collisional model, which is almost isotropic (see green line in the upper panels). The parallel correlation for this model is the largest among our collisionless models. The shortest correlation, both in the parallel and perpendicular directions, is observed in Model 4 (blue line), which is supersonic super-Alfvénic. Still, the perpendicular correlation length is almost twice as large as the parallel one for this case. Generally, for models with small $c_{\|}$(Models 3, 4 and 6), the correlation lengths in both directions are shorter, although they preserve some degree of anisotropy, and they depend on mostly on the value of $c_{\perp}$. For Models 1 and $2\left(c_{\|}=1.0\right)$, the correlation length in both directions scales with $c_{\perp}$, as well, except for Model 5, which has the largest parallel, but the shortest perpendicular correlation length among this group of models. These observation indicate that the instabilities prompted in the systems lead to more complex and smaller size configurations of the magnetic field.

\section{DISCUSSION}

\subsection{Collisionless MHD turbulence}

Concerning the turbulence cascade, two main differences can be pointed out between the collisionless and collisional MHD models.

The first is due to the differences in the phase speed of the wave modes introduced by the anisotropy in pressure. In fact, the linear Alfvén wave velocity may be strongly modified by the pressure anisotropy, and eventually become unstable (see equation 8). The concept of super/sub-Alfvénic turbulence, for instance, can now be misleading in the sense that it does not reflect anymore the dynamical importance of the magnetic field. These changes are only important in the high $\beta$ plasma regime, though. In the low $\beta$ regime, the role of the thermal pressure (and consequently of its anisotropy) is secondary in the turbulence dynamics.

Secondly, the firehose and mirror instabilities (when present) amplify the turbulent power at the small scales. This occurs in the unstable regime, in which the free energy in the pressure anisotropy (at large scales) is transformed into kinetic and magnetic fluctuations, which grow faster at small scales. The smaller the scale the more effective this energy injection is, since the instabilities have growth rates which are inversely proportional to the scale. In an evolved, saturated state, the smaller the scale the larger the local 'background' magnetic field intensity, which quenches the instabilities. As observed in Kowal et al. (2011), in order to the unstable modes to efficiently inject energy, they need to have growth times shorter than the cascading time, otherwise they are destroyed at the beginning of their development.

The interchange between thermal and mechanical energy is much more complex in the collisionless case, as non-local (in scales) energy transfer is supposed to take place. If we consider a turbulent system where the only source of energy is the mechanical energy injected at the scale $L$, the turbulent motions create anisotropies in pressure, depositing there some energy. Part of this energy is again released in mechanical form by the instabilities, mainly at the small scales, which reduces locally the pressure anisotropy. Therefore, there is transfer of mechanical energy from the large 

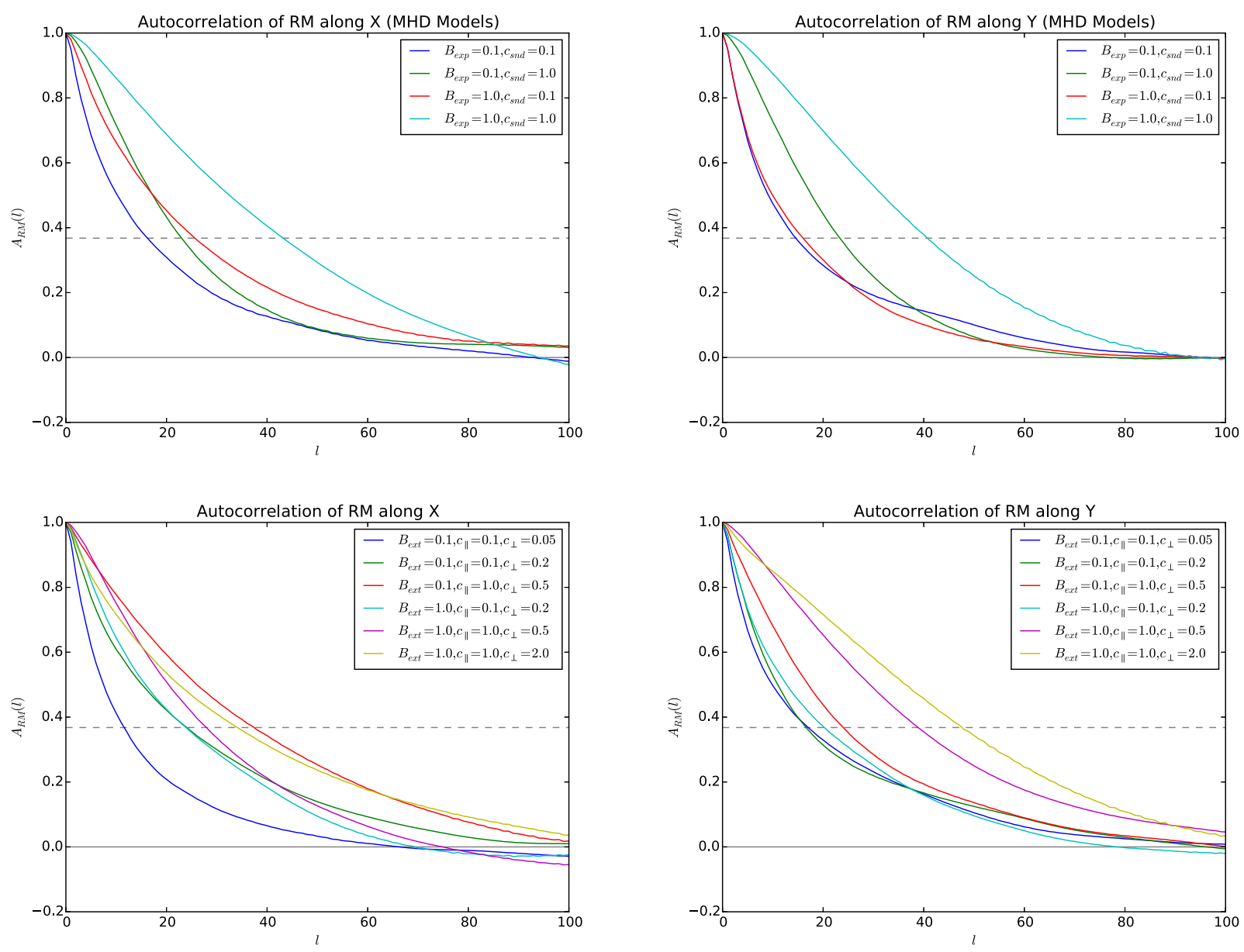

Figure 13. Autocorrelation function calculated from the Faraday Rotation maps for LoS along $\hat{x}$ and $\hat{y}$ (left- and right-hand panels, respectively) corresponding to the collisional and collisionless MHD models (upper and lower panels, respectively) as a function of the length-scale $l$.

to the small scales, which is obviously non-local. There are many pieces of evidence showing that the kinetic instabilities in the microphysical scales efficiently drain the free energy from the temperature/pressure anisotropy (caused by the large-scale fluid motions), generating entropy and quickly reducing the temperature anisotropy via anomalous collisions over all the volume (see Santos-Lima et al. 2014 , and references there). This issue, however, is still a matter of debate (see e.g. Mogavero \& Schekochihin 2014) which is beyond the scope of this work and will be discussed elsewhere.

Finally, a few considerations about dissipative effects are in order. If we completely ignore the anomalous collisions mentioned above, the physical picture of a weakly collisional plasma implies large viscosity parallel to the field lines for motions parallel to the field lines. At the same time, the other viscous components and the electric resistivity are small. ${ }^{5}$ This makes the resistive scales far below the viscous scales for the compressible motions. Based on the collisional estimates for these transport coefficients, the ICM is expected to have nearly small Reynolds numbers $(\operatorname{Re} \sim 10-100)$ but very large magnetic Reynolds numbers $\left(10^{20}\right)$, which reflects a short inertial range for the compressible motions. In this work we skipped these complications, omitting the diffusive terms in the MHD equations. However, numerical simulations always bring

${ }^{5}$ This comes from the fact that the parallel viscosity component is proportional to the ions mean free path, while the resistivity is proportional to the inverse of the mean free path for the electron-ion collisions. about effective viscosity and resistivity to the system, which should be kept in mind. While it is difficult to determine with precision the dissipative range in our simulations, we can roughly estimate it as given by scales $\lesssim 16$ cells (which for our resolution of $512^{3}$ is equivalent to $k \gtrsim 32$ ), which is in good agreement with the velocity power spectrum of the collisional MHD turbulent models presented in Kowal et al. (2011) for comparison with the collisionless models).

\subsection{The applicability of a double-isothermal closure}

The double-isothermal closure employed in this work presumes an infinite reservoir of thermal free energy $\left(p_{\|}-p_{\perp}\right)$ to be converted, by the small-scale instabilities, into mechanical and magnetic energies. This is a numerical (theoretical) approximation, with a conceptual similarity to that of the mechanical driving of turbulence. Turbulence naturally decays, but the modelling presented, in general, includes a source term that sustains the driving at large scale. Here, the instabilities naturally evolve towards a quasi-stable regime, but the numerical implementation of the double-isothermal closure corresponds, in other words, to a continuous driving of pressure anisotropy.

There is a number of physical mechanisms known to be responsible for increasing pressure anisotropy in plasmas, such as the magnetic moment conservation in expanding plasmas (Matteini et al. 2012; Falceta-Gonçalves \& Kowal 2015), beaming of particles in reconnecting magnetic fields (Gosling et al. 2005), velocity drifts 
between ions and $\alpha$-particles (Matteini et al. 2015) and others. It is very likely that at least one, and possibly more, of these mechanisms operates at large scales in the ICM. The consequence is a constant driving of pressure anisotropy, which evolves as smallscale instabilities grow. Even though the growth rate, and the saturation of instabilities to quasi-stable regimes, is known to be fast $\left(\tau_{\text {sat }}^{-1} \sim k r_{\mathrm{L}, \mathrm{i}} \Omega_{i}\right.$ ) the presence of a continuous driving source will inevitably resulting in a persistent marginally unstable regime for the plasma. The level of the pressure anisotropy will naturally depend on the anisotropy driving mechanisms and their rates. In this work this value has been conjectured, as a theoretical exercise, however the conclusions remain given that the instabilities will operate at the same scales independent on the pressure anisotropy levels.

\subsection{Faraday rotation maps as diagnostic tools}

The results of this work show that the presence of temperature/pressure anisotropies in the plasma is able to cause the development of instabilities that introduce changes in the statistics of the FR maps with respect to the standard collisional MHD. These changes are more evident in the small spatial scales (see Figs 8-10 and the power spectra in Fig. 12). Our approach can be viewed as complementary to other works, studying e.g. the $n-B$ correlation and mapping of RM as a function of radius (see Bonafede et al. 2011, 2015). Our numerical simulations provide self-consistent evolution of magnetic field and density, therefore, allow us to study the PDFs and power spectra of RM in a self-consistent way. We do not study the radial dependence of $B$ or $\rho$, however. As we explained in Section 3.3 our models represent a small box embedded in an ICM or, in other words, the small-scale part of turbulent cascade ending in the dissipation range. This, however, raises the question if these small-scale features in FR maps could be observed with the current instruments, since they could be smeared by the given beam resolution (Enßlin \& Vogt 2003). The mean free path is in range 0.05$30 \mathrm{kpc}$ for Hydra A cluster Schekochihin \& Cowley (2006), and 3-7 kpc in Coma cluster (see Andrade-Santos et al. 2013; Sanders et al. 2013). Therefore, we must consider an observational spatial resolution as fine as the order of $1 \mathrm{kpc}$. Future Square Kilometre Array (SKA) should be able to reach scales of tens of pc for the nearest clusters. Moreover, we must stress that collisionless plasma instabilities also result in modifications in the one-point statistics of FR (see Fig. 11 and Table 4), which means that a collection of a large number of measurements of distant background radio galaxies would be enough to probe this effect in a nearby cluster.

The goal of our work is to determine if the FR maps obtained from observations could manifest any sign of the collisionless plasma features, allowing us to restrict the conditions of the ICM plasma, not only the sonic and Alfvénic regimes, but also the degree of pressure anisotropy. Moreover, the FR maps provide us, under assumption of the $B-\rho$ relation, with the estimations of the strength of the magnetic field component parallel to LoS. Our studies use the exact $B-\rho$ relation, therefore allow us to test the validity of the assumptions used to determine $B$ in ICM.

Wu, Kim \& Ryu (2015) obtained an empirical law relating the magnetic field intensity in the LoS with the sonic Mach number of the turbulence and the variance of the RM measurements, which are both observable quantities. They built synthetic FR maps employing simulated collisional MHD turbulence models with different regimes of the plasma $\beta$ ratio and the sonic Mach number, for the case of solenoidal turbulence forcing, and considering different angles of view. They showed that their empirical relation could recover reasonably well the magnetic intensity for most of the LoS. In their analysis, this empirical relation may be understood in terms of the spectral distribution and the correlation of the magnetic and density fluctuations, for individual MHD modes. In the framework of the double-isothermal collisionless MHD model used in the present study (focusing on high $\beta$ turbulence aiming at applications to the ICM), the extraction of a similar empirical law would be impossible without a previous knowledge of the real anisotropy level, because this may modify drastically the MHD modes (see Figs B1 and B2 in the Appendix B) as well as the turbulence cascade (see Section 4.2).

A study of the statistics of the synthetic FR maps of collisionless models considering different closures is desired. However, such models require a subgrid approach to constrain the anisotropy level due to micro-scale processes. As remarked before, Santos-Lima et al. (2014) employed a subgrid model parametrizing the isotropization rate, in order to study the turbulent statistics and dynamo in the ICM. This may have also possible impact on the determination of the transport properties of the plasma (Schekochihin \& Cowley 2006; Santos-Lima et al. 2014; Kunz et al. 2012) and cosmic rays diffusion (through their interactions with the turbulence; (see Yan \& Lazarian 2002). The anisotropies and plasma instabilities may also have an important effect on particle acceleration by turbulence in galaxy clusters (see Brunetti \& Lazarian 2011a,b; Miniati 2015). The properties of temperature isotropization in collisionless plasmas are currently being explored in extensive theoretical and numerical studies (e.g. Schekochihin, Nazarenko \& Yousef 2012; Kunz, Schekochihin \& Stone 2014; Santos-Lima et al. 2014; Riquelme, Quataert \& Verscharen 2015). For the case of dynamo action, Bhat \& Subramanian (2013) have simulated a fluctuating dynamo with varying magnetic Reynolds numbers finding that the intermittent magnetic field contributions to the FR are still significant. Following the authors, the strong field regions contribute to only 15-20 per cent of the RM, showing that the main contribution comes from the fluctuating magnetic fields. We intend to explore this issue elsewhere.

The precise characteristics of the magnetic fields in galaxy clusters is still unknown. As we already mentioned in the beginning of this subsection, the forthcoming large radio telescopes will open a new era in the observation of these fields and should help to understand their origin and structure. The new Low Frequency Array and the planned SKA trace low-energy cosmic ray electrons allowing us to map the structure of weak magnetic fields in many regions through FR measures (Beck 2015). In this sense, the use of FR maps as diagnostic tool could lead to a better understanding and interpretation of the observations. Bonafede et al. (2015) have studied the capabilities of the SKA in constraining the properties of magnetic fields inside and around galaxy clusters. They showed that this instrument will be able to recover scales in the magnetic field properties of the ICM much smaller than the present instruments, which would be finally comparable to the simulated data available.

\section{SUMMARY AND CONCLUSIONS}

We have performed numerical simulations within two different formalisms, namely the standard collisional MHD, and a doubleisothermal collisionless MHD model that incorporates the effects of pressure anisotropy which lead to the appearance of the firehose and mirror instabilities in the dynamics of the plasma. We have analysed the magnetic field and the FR maps along different LoSs, and carried out an extensive statistical study of these two quantities including the power spectrum, the probability distribution function and two-point correlation functions. In order to better understand the 
dependence of these quantities on the turbulence regime in which the plasma evolves, we have performed the simulations for six different models covering the sub/super-Alfvénic and sub/supersonic regimes, including a model that matches the conditions prevailing in compressed regions of the ICM of galaxies.

Our results show that important imprints of the pressure anisotropy may be present in the magnetic fields and their associated FR maps. In particular, we find that the magnetic field in the collisionless MHD approach may show a more granulated structure than its MHD counterpart and its spectrum may present an excess of power at the small scales due to the enhancement of magnetic fluctuations originated from the firehose instability (which corresponds to physical scales of few to tens of kiloparsecs). This is also evidenced in the FR maps and in the correlation lengths extracted from two-point functions, which turn out to be smaller in collisionless MHD. This feature is particularly evident in the model corresponding to the conditions prevailing in compressed regions of the ICM (see Model 5 in related figures).

These imprints should be visible at small scales of the flow and may be below the limit of detectability of the current observational tools. As mentioned in Section 5.3, the SKA telescope will be able to probe such small-scale fluctuations in the ICM (Bonafede et al. 2015).

Finally, we should remark that the results above were obtained by neglecting the plasma feedback on the instabilities. Plasma particle scattering by the electromagnetic fluctuations of the instabilities may cause their saturation and the relaxation of the pressure anisotropies (Santos-Lima et al. 2014). If included in the numerical simulations, this relaxation may wash out the instabilities thus further constraining their impact on the magnetic field distribution or the FR maps. We will explore this issue in a forthcoming work.

\section{ACKNOWLEDGEMENTS}

MSN acknowledges support from a grant of the Brazilian Agency FAPESP (2010/50298-8) and from a grant of the Argentinian National Council of Scientific and Technic Investigation CONICET. GK acknowledges support from FAPESP (grants no. 2009/500538, 2013/04073-2 and 2013/18815-0) and CAPES (PNPD 1475088). EMGDP acknowledges support from FAPESP (2013/10559-5, and 2011/53275-4) and CNPq (306598/2009-4) grants RSL acknowledges support from FAPESP (2013/15115-8, and DFG thanks the European Research Council (ADG-2011 ECOGAL), and Brazilian agencies CNPq (no. 302949/2014-3), CAPES (3400-13-1) and FAPESP (no.2013/10559-5) for financial support.

\section{REFERENCES}

Andrade-Santos F., Nulsen P. E. J., Kraft R. P., Forman W. R., Jones C., Churazov E., Vikhlinin A., 2013, ApJ, 766, 107

Banerjee N., Sharma P., 2014, MNRAS, 443, 687

Barakat A. R., Schunk R. W., 1982, Plasma Phys., 24, 389

Beck R., 2015, in Lazarian A., de Gouveia Dal Pino E. M., Melioli C., eds, Astrophysics and Space Science Library, Vol. 407, Future Observations of Cosmic Magnetic Fields with LOFAR, SKA and Its Precursors. Springer-Verlag, Netherlands, p. 3

Beck R., Wielebinski R., 2013, Magnetic Fields in Galaxies. Springer Science+Business Media, Dordrecht, p. 641

Beck R., Brandenburg A., Moss D., Shukurov A., Sokoloff D., 1996, ARA\&A, 34, 155

Berezinsky V. S., Blasi P., Ptuskin V. S., 1997, ApJ, 487, 529

Bhat P., Subramanian K., 2013, MNRAS, 429, 2469
Bonafede A., Feretti L., Murgia M., Govoni F., Giovannini G., Vacca V., 2010a, preprint (arXiv:1009.1233)

Bonafede A., Feretti L., Murgia M., Govoni F., Giovannini G., Dallacasa D., Dolag K., Taylor G. B., 2010b, A\&A, 513, A30

Bonafede A., Dolag K., Stasyszyn F., Murante G., Borgani S., 2011, MNRAS, 418, 2234

Bonafede A. et al., 2015, Proc. Sci., Advancing Astrophysics with the Square Kilometre Array. Giardini Naxos, Italy, p. 95

Brandenburg A., Stepanov R., 2014, ApJ, 786, 91

Brüggen M., 2013, MNRAS, 436, 294

Brüggen M., Ruszkowski M., Simionescu A., Hoeft M., Dalla Vecchia C., 2005, ApJ, 631, L21

Brüggen M., Bykov A., Ryu D., Röttgering H., 2012, Space Sci. Rev., 166, 187

Brunetti G., Jones T. W., 2014, Int. J. Modern Phys. D, 23, 30007

Brunetti G., Lazarian A., 2007, MNRAS, 378, 245

Brunetti G., Lazarian A., 2011a, MNRAS, 410, 127

Brunetti G., Lazarian A., 2011b, MNRAS, 412, 817

Carilli C. L., Taylor G. B., 2002, ARA\&A, 40, 319

Cavaliere A., Fusco-Femiano R., 1978, A\&A, 70, 677

Chew G. F., Goldberger M. L., Low F. E., 1956, R. Soc. London Proc. Ser. A, 236, 112

Cho J., Lazarian A., 2002, Phys. Rev. Lett., 88, 245001

Clarke T. E., Kronberg P. P., Böhringer H., 2001, ApJ, 547, L111

de Gouveia Dal Pino E. M., 2010, preprint (arXiv:1003.3884)

Dolag K., 2006, Astron. Nachr., 327, 575

Dolag K., Bartelmann M., Lesch H., 2002, A\&A, 387, 383

Dolag K., Grasso D., Springel V., Tkachev I., 2005, J. Cosmol. Astropart. Phys., 001, 009

Enßlin T. A., Vogt C., 2003, A\&A, 401, 835

Falceta-Gonçalves D., Kowal G., 2015, ApJ, 808, 65

Feretti L., Giovannini G., Govoni F., Murgia M., 2012, A\&AR, 20, 54

Ferrari C., Govoni F., Schindler S., Bykov A. M., Rephaeli Y., 2008, Space Sci. Rev., 134, 93

Fletcher A., 2010, in Kothes R., Landecker T. L., Willis A. G., eds, ASP Conf. Ser. 438, The Dynamic Interstellar Medium: A Celebration of the Canadian Galactic Plane Survey. Astron. Soc. Pac., San Francisco, p. 197

Gonzalez A. H., Zaritsky D., Zabludoff A. I., 2007, ApJ, 666, 147

Gosling J. T., Skoug R. M., McComas D. J., Smith C. W., 2005, J. Geophys. Res. (Space Phys.), 110, 1107

Govoni F., Feretti L., 2004, Int. J. Mod. Phys. D, 13, 1549

Govoni F. et al., 2010, A\&A, 522, A105

Hau L.-N., Wang B.-J., 2007, Nonlinear Proc. Geophys., 14, 557

Iapichino L., Brüggen M., 2012, MNRAS, 423, 2781

Jones T. W., Porter D. H., Ryu D., Cho J., 2011, Mem. Soc. Astron. Italiana, 82,588

Kowal G., Lazarian A., 2010, ApJ, 720, 742

Kowal G., Lazarian A., Beresnyak A., 2007, ApJ, 658, 423

Kowal G., Lazarian A., Vishniac E. T., Otmianowska-Mazur K., 2009, ApJ, 700,63

Kowal G., Falceta-Gonçalves D. A., Lazarian A., 2011, New J. Phys., 13, 053001

Krall N. A., Trivelpiece A. W., 1973, Principles of Plasma Physics. McGrawHill Inc., US

Kuchar P., Enßlin T. A., 2011, A\&A, 529, A13

Kulsrud R. M., 1983, in Galeev A. A., Sudan R. N., eds, Basic Plasma Physics: Selected Chapters, Handbook of Plasma Physics, Vol. 1 MHD Description of Plasma, North-Holland Publishing Company, Amsterdam, p. 1

Kunz M. W., 2011, MNRAS, 417, 602

Kunz M. W., Bogdanović T., Reynolds C. S., Stone J. M., 2012, ApJ, 754, 122

Kunz M. W., Schekochihin A. A., Stone J. M., 2014, Phys. Rev. Lett., 112, 205003

Lapi A., Cavaliere A., Fusco-Femiano R., 2012, ApJ, 745, L15

Lazarian A., 2006, ApJ, 645, L25 
Markevitch M., Govoni F., Brunetti G., Jerius D., 2005, ApJ, 627, 733

Matteini L., Hellinger P., Landi S., Trávníček P. M., Velli M., 2012, Space Sci. Rev., 172, 373

Matteini L., Hellinger P., Schwartz S., Landi S., 2015, ApJ, 812, 13

Miniati F., 2015, ApJ, 800, 60

Mogavero F., Schekochihin A. A., 2014, MNRAS, 440, 3226

Murgia M., Govoni F., Feretti L., Giovannini G., Dallacasa D., Fanti R., Taylor G. B., Dolag K., 2004, A\&A, 424, 429

Nakwacki M. S., Peralta-Ramos J., 2012, J. Phys. Conf. Ser., 370, 012038

Narayan R., Medvedev M. V., 2001, ApJ, 562, L129

Petrosian V., 2001, ApJ, 557, 560

Quest K. B., Shapiro V. D., 1996, J. Geophys. Res., 101, 24457

Riquelme M. A., Quataert E., Verscharen D., 2015, ApJ, 800, 27

Sanders J. S., Fabian A. C., Churazov E., Schekochihin A. A., Simionescu A., Walker S. A., Werner N., 2013, Science, 341, 1365

Santos-Lima R., de Gouveia Dal Pino E. M., Kowal G., Falceta-Gonçalves D., Lazarian A., Nakwacki M. S., 2014, ApJ, 781, 84

Schekochihin A. A., Cowley S. C., 2006, Phys. Plasmas, 13, 056501

Schekochihin A. A., Nazarenko S. V., Yousef T. A., 2012, Phys. Rev. E, 85, 036406

Sofue Y., Fujimoto M., Wielebinski R., 1986, ARA\&A, 24, 459

Subramanian K., Shukurov A., Haugen N. E. L., 2006, MNRAS, 366 , 1437

van Weeren R. J., Brüggen M., Röttgering H. J. A., Hoeft M., 2011, MNRAS, 418,230

Vogt C., Enßlin T. A., 2005, A\&A, 434, 67

Wu Q., Kim J., Ryu D., 2015, New Astron., 34, 21

Yan H., Lazarian A., 2002, Phys. Rev. Lett., 89, 1102

\section{APPENDIX A: FIREHOSE AND MIRROR INSTA B IL I T IES}

The linear analysis of the double-isothermal collisionless MHD equations (see equations 3 to 8 ) reveals that they allow for the occurrence of the firehose and mirror instabilities which are described below.

A0.1 Firehose instability $\left(\mathrm{c}_{\|}>\mathrm{c}_{\perp}\right)$

Defining:

$f=c_{\perp}^{2} / c_{\|}^{2}-1+V_{\mathrm{A}}^{2} / c_{\|}^{2}$.

When $f<0$, the Alfvén modes become unstable and there is no wave propagation. This happens because the tension force resisting to the bending of the field lines disappears The growth rate of the firehose instability associated to this unstable Alfvén modes is given by

$\left(\gamma_{\mathrm{f}, \mathrm{A}}\right)^{2}=c_{\|}^{2}|f| k^{2} \cos ^{2} \theta$,

showing that the fastest growing mode is parallel to the background magnetic field $(\theta=0)$.

For specific angles of propagation, the slow modes also become unstable for $f<0$. These angles are in the interval:

$0<\theta<\arccos \sqrt{1+f /\left(1-c_{\perp}^{4} / c_{\|}^{4}\right)}$,

and the maximum growth rate will be for the mode parallel to the background magnetic field (i.e. $\theta=0$, which is in the limit of incompressible pseudo-Alfvén modes):

$\theta_{\max }=0, \quad\left(\gamma_{\mathrm{f}, \mathrm{S}}\right)_{\max }^{2}=c_{\|}^{2}|f| k^{2}$.

A0.2 Mirror instability $\left(\mathrm{c}_{\perp}>\mathrm{c}_{\|}\right)$

We define:

$m=-c_{\perp}^{2} / c_{\|}^{2}+1+V_{\mathrm{A}}^{2} / c_{\perp}^{2}$.

In the regime $m<0$ (which implies $c_{\perp}>c_{\|}$), the slow waves can become unstable for some propagating angles, giving rise to the mirror modes. The unstable modes have angles in the interval

$\arccos \sqrt{\max \left[1,-\frac{1}{2} m\left(c_{\|}^{2} / c_{\perp}^{2}\right) /\left(1-c_{\|}^{4} / c_{\perp}^{4}\right)\right]}<\theta<\pi / 2$.

The maximum growth rate will be given by

(i) $0>m>-2\left(1-c_{\|}^{4} / c_{\perp}^{4}\right)\left(c_{\perp}^{2} / c_{\|}^{2}\right)$

$\theta_{\max }=\arccos \sqrt{-\frac{1}{2} m\left(c_{\|}^{2} / c_{\perp}^{2}\right) /\left(1-c_{\|}^{4} / c_{\perp}^{4}\right)}$,

$\left(\gamma_{m, \mathrm{~S}}\right)_{\max }^{2}=\frac{k^{2}}{2}\left\{\sqrt{\left(c_{\perp}^{2}+V_{\mathrm{A}}^{2}\right)^{2}+c_{\|}^{4} m^{2} /\left(1-c_{\|}^{4} / c_{\perp}^{4}\right)}\right\}-$

$$
-\left(c_{\perp}^{2}+V_{\mathrm{A}}^{2}\right) \frac{k^{2}}{2}
$$

(ii) $m<-2\left(1-c_{\|}^{4} / c_{\perp}^{4}\right)\left(c_{\perp}^{2} / c_{\|}^{2}\right)$

$\theta_{\max }=0$,

$\left(\gamma_{m, \mathrm{~S}}\right)_{\max }^{2}=\frac{1}{2}\left\{\left|2 c_{\|}^{2}-c_{\perp}^{2}-V_{\mathrm{A}}^{2}\right|-\left(c_{\perp}^{2}+V_{\mathrm{A}}^{2}\right)\right\} k^{2}$.

The mirror modes conserve the conventional property of the slow modes, i.e. they allow for a negative correlation between the density fluctuations and the magnetic field component parallel to the background magnetic field.

\section{APPENDIX B: LINEAR DISPERSION FOR THE INITIAL CONDITIONS OF THE MODELS}

Fig. B1 shows the real and imaginary phase velocities of the linear waves as a function of the propagation angle (see dispersion relation in equation 8) for the initial conditions of each of the simulated models in this work (see Table 1).

Fig. B2 depicts the density-magnetic field fluctuation correlation for the compressible linear modes as a function of the propagation angle, also for the initial conditions of each model of Table 1. This correlation was calculated using equation (9) of Hau \& Wang (2007):

$r_{\mathrm{F}, \mathrm{S}} \equiv\left(\frac{\delta B}{\delta \rho}\right)_{\mathrm{F}, \mathrm{S}}\left(\frac{B_{\mathrm{ext}}}{\rho_{0}}\right)=\frac{a \pm \sqrt{\Delta}}{2\left(a+c_{\perp}^{2}\right)}$,

where $a=V_{\mathrm{A}}^{2}-c_{\perp}^{2}+2\left(c_{\perp}^{2}-c_{\|}^{2}\right) \cos ^{2} \theta$, the \pm are for the fast (F) and slow (S) modes, and $\Delta$ is given by equation (10). 
Model 1

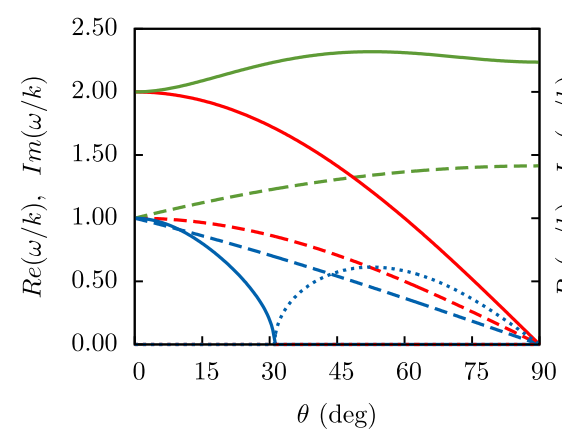

Model 4

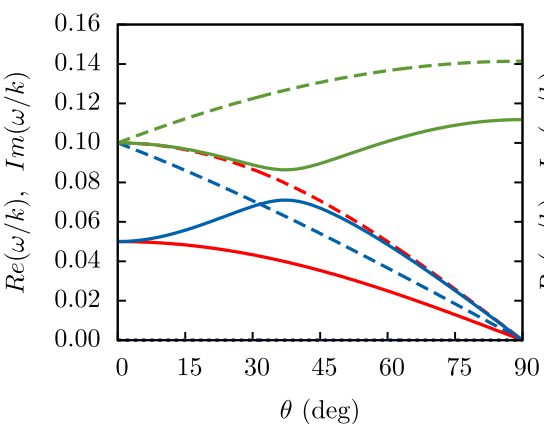

Model 2

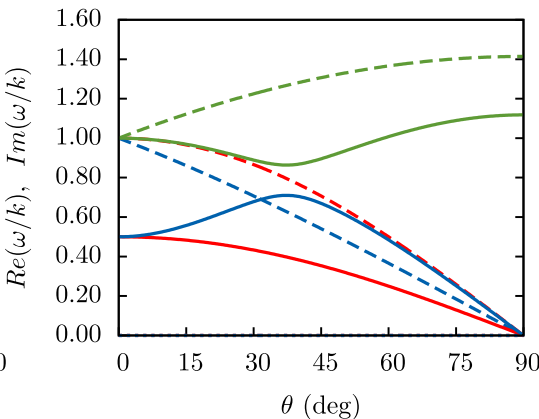

Model 5

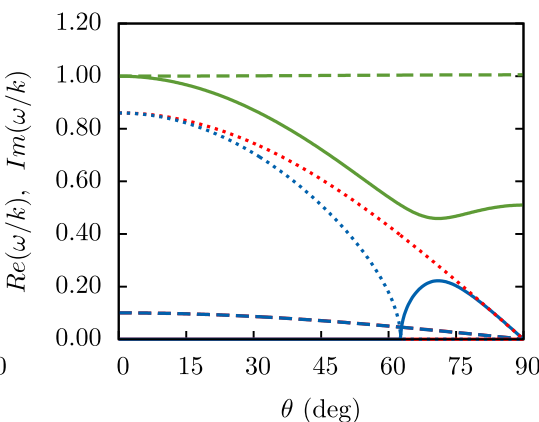

Model 3

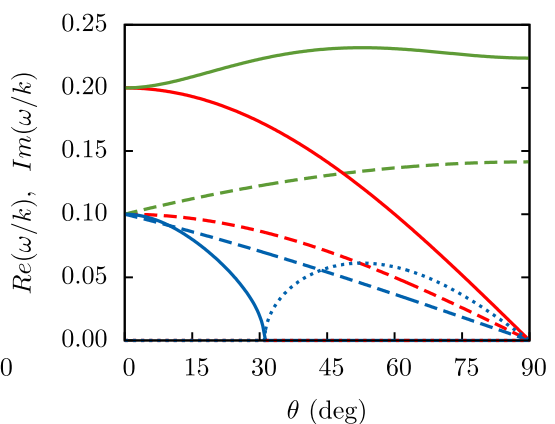

Model 6

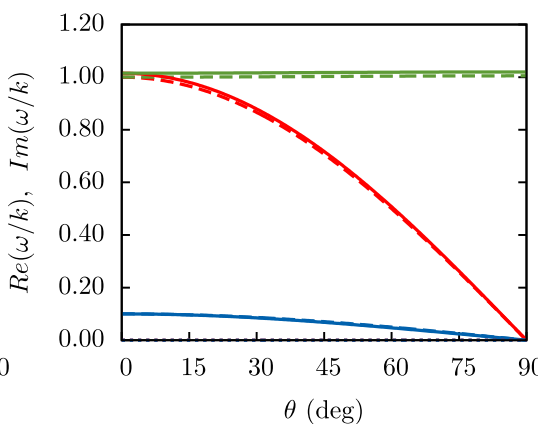

Figure B1. Real (continuous lines) and imaginary (dotted lines) linear phase velocities for different waves considering the initial conditions of the simulated models (see Table 1). The phase speeds for the comparative MHD models are given by dashed lines. Each colour represents a different wave: Alfvén (red), fast (green) and slow magnetosonic (blue).

Model 1

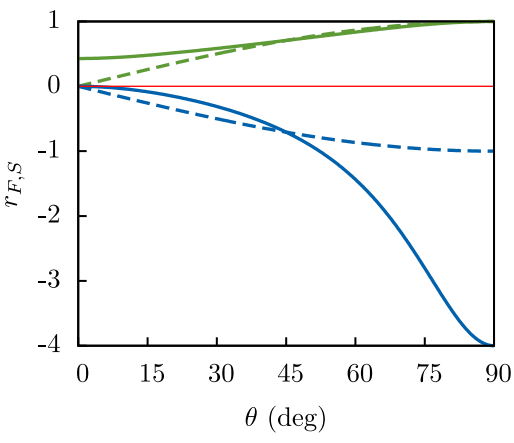

Model 4

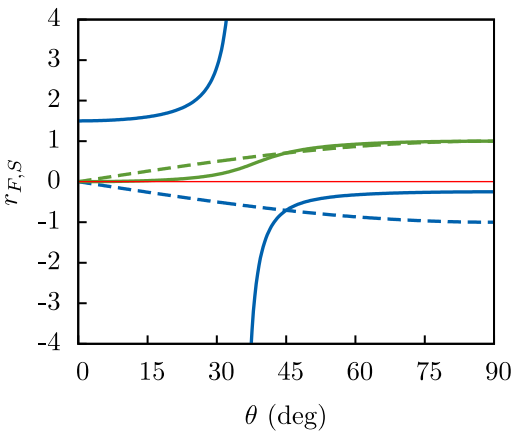

Model 2

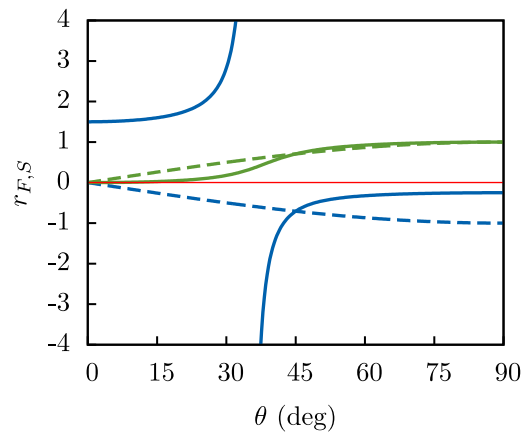

Model 5

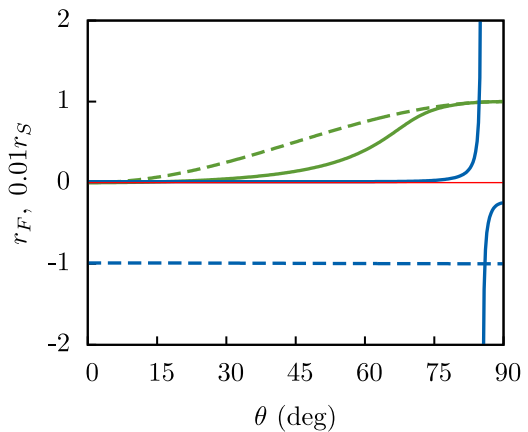

Model 3

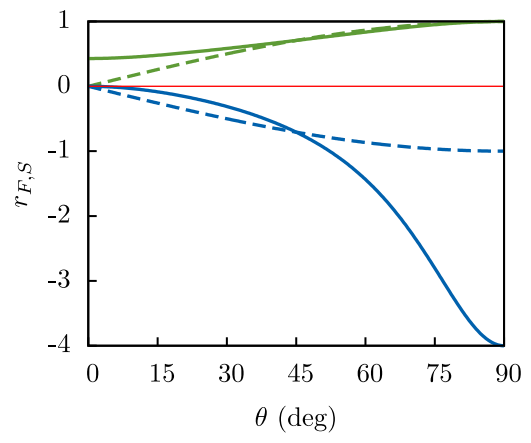

Model 6

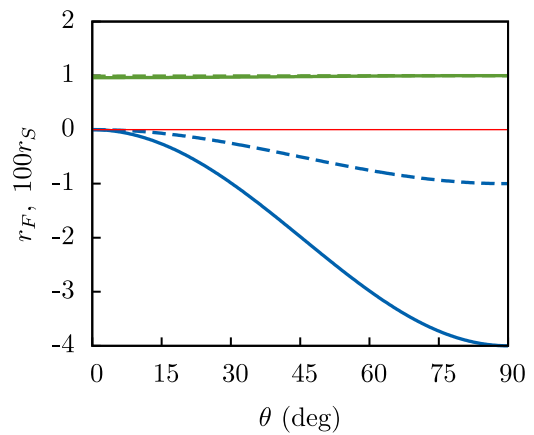

Figure B2. The lines show the magnetic-density correlation (equation B1) for the linear modes slow (blue) and fast (green) as a function of the propagation angle, calculated for the initial conditions of the simulated models. The solid lines represent the anisotropic pressure collisionless models and the dashed lines represent the comparative collisional MHD models (see Table 1).

This paper has been typeset from a $\mathrm{T}_{\mathrm{E}} \mathrm{X} / \mathrm{E} \mathrm{T} \mathrm{E}$ file prepared by the author. 